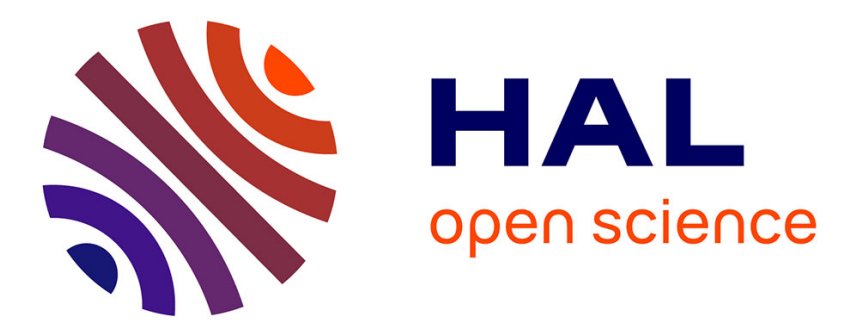

\title{
Line broadening coefficient calculations for methane perturbed by nitrogen
}

Tony Gabard, Vincent Boudon

\section{To cite this version:}

Tony Gabard, Vincent Boudon. Line broadening coefficient calculations for methane perturbed by nitrogen. Journal of Quantitative Spectroscopy and Radiative Transfer, 2010, 111 (10), pp.1328-1343. 10.1016/j.jqsrt.2010.01.032 . hal-00452656

\section{HAL Id: hal-00452656 https://hal.science/hal-00452656}

Submitted on 2 Feb 2010

HAL is a multi-disciplinary open access archive for the deposit and dissemination of scientific research documents, whether they are published or not. The documents may come from teaching and research institutions in France or abroad, or from public or private research centers.
L'archive ouverte pluridisciplinaire HAL, est destinée au dépôt et à la diffusion de documents scientifiques de niveau recherche, publiés ou non, émanant des établissements d'enseignement et de recherche français ou étrangers, des laboratoires publics ou privés. 


\title{
Line broadening coefficient calculations for methane perturbed by nitrogen
}

\author{
Tony Gabard ${ }^{\mathrm{a}, 1, *}$, Vincent Boudon ${ }^{\mathrm{a}, 1}$ \\ ${ }^{a}$ Laboratoire Interdisciplinaire Carnot de Bourgogne, UMR CNRS 5209, Université de \\ Bourgogne, 9 Av. A. Savary, BP 47870, F-21078 Dijon, France
}

\begin{abstract}
We report semiclassical line broadening calculations for methane perturbed by nitrogen at room temperature. For this, we have developed a symmetrized version of the Robert and Bonamy theory. The interaction potential was built from electrostatic (octopole and hexadecapole for methane, quadrupole for nitrogen) and atom-atom contributions. The relative (classical) trajectories of the molecules were computed in the frame of the usual parabolic model, through analytical formula. High orders of developments had to be used for the short range molecular interactions. As a consequence, a combination of symbolic computation and source code generation was employed to implement practical calculations. We have compared our calculations to the most recent experimental data available in the $\nu_{4}$ band, the $\nu_{3}$ band and the octad spectral region of methane.
\end{abstract}

Keywords: Methane, Nitrogen, Infrared spectroscopy, Line broadening coefficients

PACS: 33.20.Ea, 33.70.Jg, 34.10.+x, 34.50.Ez

\section{Introduction}

Besides being one of the major greenhouse gases on Earth [1, 2], methane $\left(\mathrm{CH}_{4}\right)$ is an important molecule for many planetary atmospheres, either in the Solar System (Mars [3], Jupiter [4, 5], Saturn [6] and its main satellite

\footnotetext{
*Corresponding author. Fax: +33 380395971

Email addresses: Tony.Gabard@u-bourgogne.fr (Tony Gabard), Vincent.Boudon@u-bourgogne.fr (Vincent Boudon)

${ }^{1}$ http://icb.u-bourgogne.fr/OMR/SMA/
} 
Titan [7-11], Uranus [12], Neptune [13] and its main satellite Triton [14], Pluto $[14,15])$ or also farther away, since it has now been identified in the gas envelope of at least two giant extrasolar planets (exoplanets HD 189733b [16] and HD 209458b [17]). In some cases, the presence of methane rises important questions about its origin (Mars [18, 19], Titan [20], exoplanets, ...) or leads to very interesting chemical processes (Titan [21]). Spectroscopy being the best (if not the sole) mean for studying such distant atmospheres, it is thus essential to have an accurate model of methane's absorption spectrum over a wide spectral range, from microwave to visible regions. In short, this is done by recording laboratory high-resolution spectra, then by analyzing them in order to get molecular (Hamiltonian, dipole moment and lineshape) parameters allowing to create detailed linelists. These linelists are then used in atmospheric radiative transfer models. The methane spectrum, however, is quite complicated and thus it is still the subject of many experimental and theoretical efforts. The vibrational structure is organized into polyads of rapidly increasing complexity when climbing the energy range up to midinfrared and visible regions [22]. As far as line positions and intensities are concerned, the most recent and complete global analysis for ${ }^{12} \mathrm{CH}_{4}$ concerns the 0 to $4800 \mathrm{~cm}^{-1}$ region [23], while upper polyads are still under investigation and the subject of sophisticated experimental efforts [24-28].

One object of particular interest in the Solar System is Titan. Its nitrogen atmosphere contains significant amounts of methane that lead to a rich organic chemistry [21], as well as to a fascinating "methane cycle" [29]. Due to this large quantity of $\mathrm{CH}_{4}$ in a dense atmosphere (which is also the case for giant planets and exoplanets), it is necessary to model methane's absorption spectrum up to highly excited polyads. In addition to positions and intensities, it is clear that collisional broadening and line-mixing effects on lineshapes is an essential aspect to address in order to develop an accurate model. This question is a very difficult one, especially because of the physical conditions on Titan. In short, near the ground in Titan's troposphere, the pressure is 1.5 bar and the temperature is around $80 \mathrm{~K}$ for $c a .4 \%$ of $\mathrm{CH}_{4}$ in $\mathrm{N}_{2}$. Methane contributes to the opacity of Titan's atmosphere up to the visible regions. Presently, the high energy polyads for which no detailed analysis exists are handled in the atmospheric calculations through low-resolution empirical "band models" $[9,30]$ which are not very satisfactory as they do not allow to account for collisional broadening effects. Solving this problem will thus possibly require many years of experimental and theoretical efforts.

This paper specifically concentrates on collisional broadening coefficient 
calculations for methane perturbed by nitrogen. Before investigating the low temperatures conditions at high wavenumbers described above, we first considered here room temperature collisional parameters in the lowest polyads of methane. The subject of infrared line shapes studies for methane is extensively documented from an experimental point of view. Some of the most recent works dealt with measurements in the first four polyads of methane (dyad near $1300 \mathrm{~cm}^{-1}$, pentad near $3000 \mathrm{~cm}^{-1}$, octad near $4300 \mathrm{~cm}^{-1}$ and tetradecad near $6000 \mathrm{~cm}^{-1}$ ) [31-43]. We note that the temperature dependencies of line shape parameters and/or line mixing parameters could be determined in some of these studies. A review of earlier experimental studies may be found in Ref. [44]. Here, we will be concerned by a model for the calculations of broadening coefficients for individual spectral lines. Depending on the thermodynamic conditions in the medium, e.g. those of Titan's atmosphere, the correct modeling of line positions, intensities and broadenings will certainly not suffice. Thus, the present model will have to be extended so that to include line-mixing (collisional interferences between spectral lines) $[6,45-53]$.

\section{Theoretical elements}

\subsection{Energies and eigenfunctions for methane}

These quantities are obtained through the diagonalization of an effective Hamiltonian built from elementary rotational and vibrational operators. The related symmetry adapted tensorial formalism has been extensively described, e.g. in Refs. [54, 55], and references cited therein. Thus, we only give the meanings of some of the notations that will be used in section 2.2. Eigenstates are labeled with $P, J, C$ and $\alpha$ (also denoted as $n$ or $N$ in some references).

$P$ is the polyad number. The ground state, the dyad, the pentad, the octad and the tetradecad have numbers $0,1,2,3$ and 4, for example. Since, in the following, we will use the labels $i$ and $f$ to denote the lower and upper states of a given methane transition, we will omit the (implicit) polyad number.

$J$ is the total angular momentum. It is conventionally of $g$ parity (in the rotation group related to the construction of rotational operators and rotational basis functions for the Hamiltonian). 
$C$ is the symmetry label in the $T_{d}$ point group $\left(A_{1}, A_{2}, E, F_{1}\right.$ and $\left.F_{2}\right)$.

$\alpha, n$ or $N$ is usually called the order index. It numbers the energies in increasing values inside an Hamiltonian block, after diagonalization, at fixed $P, J$ and symmetry $C$.

Eigenfunctions are expressed in terms of basis functions through a unitary transformation,

$$
\begin{aligned}
& \left|J_{g} C \alpha \sigma\right\rangle= \\
& \quad=\sum_{n_{r}, C_{r},\left\{v_{s}\right\}, C_{v}} U_{n_{r}, C_{r},\left\{v_{s}\right\}, C_{v}}^{(J, C, \alpha}\left|J_{g} n_{r} C_{r} ;\left\{v_{s}\right\} C_{v} ; C \sigma\right\rangle
\end{aligned}
$$

where $n_{r}, C_{r}$ are the labels for the rotational basis functions and $\left\{v_{s}\right\}, C_{v}$ are the labels for the vibrational basis functions. More details are given in Ref. [56]. $\sigma$ is the component index for symmetry $C$ (in case it is degenerate). For simplicity, we will omit the implicit $g$ parity index for the angular momentum of the eigenfunctions in what follows.

\subsection{Line broadening theory}

More general developments on line shapes theories, including semiclassical approaches are described in Ref. [57]. Here, we are using a semiclassical theory originally developed by Robert and Bonamy [58] for linear molecules. Since that time, numerous applications have been reported. In some of the most recent works, this theory was applied to non linear molecules, e.g. water [59] or nitric acid [60]. In the latest application of this theory to the case of methane perturbed by nitrogen [44], a classical formalism was used to describe the wavefunctions of the active molecule. It is classical in the sense that methane eigenfunctions were developed in terms of rotation group basis functions. Thus, it was necessary to apply a basis transformation to symmetry adapted wavefunctions (in the $T_{d}$ point symmetry group) in order to get wavefunctions suitable for line broadening calculations. As a consequence, this procedure is restricted to methane vibrational bands that admit a tensorial extension to the full rotation group. This is approximatively the case for the $\nu_{3}$ band if we consider it as isolated from other vibrational bands in the pentad region.

As an alternative to this approach, we have implemented a line broadening theory that makes full use of the tetrahedral formalism [54, 55], consistent with the theory used for infrared line positions and intensities calculations 
of methane. This way, calculations may be done for any vibrational bands of methane, using directly energies and eigenfunctions as obtained from accurate vibrational polyads analysis of this molecule [23] (and references cited therein). Thus, the present work comes as an extension of the theory that we have developed for methane perturbed by a rare gas [61-63], where we used the same symmetry adaptation for methane. An important feature of these theories is that when developing the intermolecular potential for methane perturbed by any kind of molecule in terms of radial and angular functions, then the angular operators related to methane are all of definite symmetries in the $T_{d}$ group, i.e. either $A_{1}$ or $A_{2}$ [61]. This property allows to use directly the methane wavefunctions, as given by Eq. (1), in line broadening calculations, as described below.

For a methane line with lower state $i\left(\equiv\left\{J_{i}, C_{i}, \alpha_{i}, \sigma_{i}\right\}\right)$ and upper state $f\left(\equiv\left\{J_{f}, C_{f}, \alpha_{f}, \sigma_{f}\right\}\right)$, the line broadening coefficient, in units of $\mathrm{cm}^{-1} \cdot \mathrm{atm}^{-1}$, is expressed as

$$
\begin{aligned}
& \gamma_{f i}=\frac{1}{2 \pi c} L \frac{T_{0}}{T P_{0}} \int_{0}^{\infty} d v v f_{B}(v) \sum_{\{2\}} \rho_{\{2\}} \times \\
& \times \int_{r_{c_{0}}}^{\infty} 2 \pi r_{c} d r_{c}\left(\frac{v_{c}^{\prime}}{v}\right)^{2}\left[1-\exp \left(-S_{2, f i,\{2\}}\left(r_{c}, v\right)\right)\right]
\end{aligned}
$$

where $c$ is the speed of light, $L=2.6867775 \times 10^{19} \mathrm{~cm}^{-3}$ is Loschmidt's number, $T$ is the temperature, $T_{0}$ and $P_{0}$ are the temperature and pressure at $\mathrm{STP}$ conditions, $v$ is the relative velocity modulus for the methane-nitrogen couple, $f_{B}(v)$ is the Boltzmann distribution function for $v,\{2\}$ represents all the labels for a given perturber quantum state, $\rho_{\{2\}}$ is the population factor for such a state. $r_{c}$ is the distance between the centers of mass of the molecules at the time of closest approach for a given impact parameter. $v_{c}^{\prime}$ is the apparent relative velocity at that time. $r_{c_{0}}$ is the minimum value of $r_{c}$, i.e. the one at the classical turning point of the relative trajectory governed by the isotropic interaction potential. $S_{2, f i,\{2\}}$ results from a second order expansion of the scattering operator and contains, schematically, sums of probabilities of collisions that induce changes of quantum states from the lower state $i$ and the upper state $f$ of the considered methane line. In practice, we replace the integration over the relative velocity distribution by a calculation at the mean thermal velocity. Thus, we set

$$
f_{B}(v)=\delta(v-\bar{v}), \text { with } \bar{v}=\left(\frac{8 K_{B} T}{\pi \mu}\right)^{1 / 2}
$$


where $K_{B}$ is Boltzmann's constant and $\mu$ is the reduced mass of the molecular couple.

The $S_{2, f i,\{2\}}$ term in Eq. (2) contains the usual "outer" and "middle" contributions $[58,64]$. As an example, inelastic contributions that affect the upper state of the transition are accounted for through $S_{2, f,\{2\}}$,

$$
\begin{aligned}
& S_{2, f,\{2\}}=\frac{\hbar^{-2}}{2\left[J_{f}, C_{f}, J_{2}\right]} \sum_{J_{f^{\prime}}, C_{f^{\prime}}, \alpha_{f^{\prime}}} \sum_{J_{2^{\prime}}} \sum_{l_{1}, l_{2}}\left[l_{1}, l_{2}\right]^{-1} \times \\
& \times \sum_{m_{1}, m_{2}}\left|(F . T .)_{m_{1} m_{2}}^{\left(l_{1}, \Gamma_{1} ; l_{2}\right)}\left(f, f^{\prime}, 2,2^{\prime}\right)\right|^{2} \times \\
& \times \mid\left.(\text { M.M.E. })^{\left(l_{1}, \Gamma_{1}\right)}\left(f, f^{\prime}\right)\right|^{2} \times \\
& \times(\text { P.S.M.E. })^{\left(l_{2}\right)}\left(2,2^{\prime}\right)
\end{aligned}
$$

The notation $[I]$ stands for the dimension of the irreducible representation $I$ of a group. $[J($ or $l)]=2 J($ or $l)+1$ and $[C]=1,1,2,3$ and 3 for $C=A_{1}, A_{2}$, $E, F_{1}$ and $F_{2}$, respectively. $l_{1}$ and $l_{2}$ are the tensorial ranks of the angular operators of the anisotropic interaction potential for methane and nitrogen, respectively. $m_{1}$ and $m_{2}$ are the associated projection numbers. $\Gamma_{1},=A_{1}$ or $A_{2}$, is the $T_{d}$ group symmetry label attached to $l_{1}$. In Eq. (4), the (F.T.) are Fourier Transforms,

$$
\begin{aligned}
& (F . T .)_{m_{1} m_{2}}^{\left(l_{1}, \Gamma_{1} ; l_{2}\right)}\left(f, f^{\prime}, 2,2^{\prime}\right)= \\
& =\int_{-\infty}^{\infty} d t e^{i\left(\omega_{f, f^{\prime}}+\omega_{2,2^{\prime}}\right) t} \sum_{m} V_{|m|}^{l_{1}, \Gamma_{1}, l_{2}}(R(t)) \times \\
& \quad \times \mathcal{D}_{m_{1} m}^{\left(l_{1}\right)}(\Omega(t)) \mathcal{D}_{m_{2}-m}^{\left(l_{2}\right)}(\Omega(t))
\end{aligned}
$$

These Fourier transforms are done along the classical relative trajectories described by $R(t)$, the distance between the centers of mass of the molecules, and by $\Omega(t)$, the angle between the intermolecular axis and the $z$ axis of a fixed frame. The $\omega_{f, f^{\prime}}$ and $\omega_{2,2^{\prime}}$ are pulsations associated to the energy spacings between states $f$ and $f^{\prime}$ for methane and states 2 and $2^{\prime}$ for nitrogen, respectively. The $V$ are radial functions of the anisotropic interaction potential and the $\mathcal{D}$ 's are Wigner functions.

The $V$ radial functions are given by

$$
\begin{aligned}
& V_{|m|}^{l_{1}, \Gamma_{1}, l_{2}}(R(t))={ }_{|m|} f^{l_{1}, l_{2}} \frac{F^{l_{1}, l_{2}}}{R(t)^{l_{1}+l_{2}+1}}+ \\
& +\sum_{q}\left[{ }_{|m|} d_{q}^{l_{1}, l_{2}} \frac{D_{q}^{l_{1}, l_{2}}}{R(t)^{12+q}}-{ }_{|m|} e_{q}^{l_{1}, l_{2}} \frac{E_{q}^{l_{1}, l_{2}}}{R(t)^{6+q}}\right]
\end{aligned}
$$


The notation $|m|$ means that the expressions depend only on the absolute values of the $m$ 's projection numbers. The terms with the $f$ and $F$ factors in this equation account for the electrostatic interactions between the molecules. The $f$ are numerical coefficients. The $F$ depend on the multipolar moments of the molecules, i.e. octopole and hexadecapole for methane and quadrupole for nitrogen. The terms with the $d, D, e$ and $E$ factors are the atom-atom short range contributions to the potential. They result from two center expansions of sums of Lennard-Jones interactions between the atoms of the molecules. The $d$ and $e$ are numerical coefficients. The $D$ and $E$ are polynomials depending on the atom-atom interaction parameters and on some geometrical parameters of the two molecules. Detailed expressions for the above factors are not given here but may be found in Ref. [56]. For this potential, the maximum value of $l_{1}+l_{2}$ defines the order of development for angular functions. Similarly, the maximum value of $q$ defines the order of development for the radial functions. The calculations of this paper were done with $l_{1}+l_{2} \leq 6$ (fixed) and $q_{\max }=14$, to ensure proper convergence. It means, for the angular operators, that the sets of labels that appear in the development are $\left\{\left(l_{1}, \Gamma_{1}\right) ; l_{2}\right\}=\left\{\left(0, A_{1}\right) ; 0\right\}$ (short range, isotropic), $\left\{\left(0, A_{1}\right) ; 2\right\},\left\{\left(0, A_{1}\right) ; 4\right\},\left\{\left(0, A_{1}\right) ; 6\right\},\left\{\left(3, A_{2}\right) ; 0\right\},\left\{\left(3, A_{2}\right) ; 2\right\}$ (short range and electrostatic), $\left\{\left(4, A_{1}\right) ; 0\right\},\left\{\left(4, A_{1}\right) ; 2\right\}$ (short range and electrostatic) and $\left\{\left(6, A_{1}\right) ; 0\right\}$. The six sets with no indications correspond to short range interaction contributions.

The (M.M.E.) in Eq. (4) are Methane (doubly reduced) Matrix Elements,

$$
\begin{aligned}
& \text { (M.M.E. })^{\left(l_{1}, \Gamma_{1}\right)}\left(f, f^{\prime}\right)= \\
& =\left\langle J_{f} ; J_{f} C_{f} \alpha_{f}\left\|\mathcal{D}^{\left(l_{1 g}, l_{1 g}, 0 \Gamma_{1}\right)}\right\| J_{f^{\prime}} ; J_{f^{\prime}} C_{f^{\prime}} \alpha_{f^{\prime}}\right\rangle
\end{aligned}
$$

Note that we use a double tensor notation since, in the derivation of the theory, we need to make reference to the intermolecular frame (the one with its $z$ axis along the line joining the centers of mass of the two molecules) and the molecule fixed frame. The $\mathcal{D}$ are symmetrized double tensors (of symmetry $\Gamma_{1}$ ) associated to the angular operators of the interaction potential related to the active molecule. Their expressions were given previously [61].

We note that the multiplicity index of the decomposition of the $l_{1 g}$ irreducible representation of $O(3)$ into representations of $T_{d}$ is always 0 (the one besides " $\Gamma_{1}$ " in the above equation). It means that $\Gamma_{1}$, either $A_{1}$ or $A_{2}$ as stated above, appears only once in the decomposition up to $l_{1}=8$. There are some other point symmetry groups, like $C_{3 v}$, for which it is not the case [65]. 
As a consequence, expressions like Eq. (4) should not be directly applied to such groups and thus must be rederived.

To obtain the matrix elements of Eq. (7), we have derived a very general theorem for any multipolar transition moments, possibly induced by the vibration and the rotation of the methane molecule. These transition operators are written as

$$
\left[\mathcal{D}^{\left(l_{g}, l_{g}, n_{c} \Gamma_{c}\right)} \times\left(R^{\Omega\left(K_{g}, N \Gamma_{r}\right)} \times{ }^{\epsilon} V_{\{n\}\left\{n^{\prime}\right\}}^{\Gamma_{n} \Gamma_{n^{\prime}}\left(\Gamma_{v}\right)}\right)^{\left(\Gamma_{o}\right)}\right]^{(\Gamma)}
$$

where $\mathcal{D}$ is a multipole direction cosine, $R$ is a rotational operator and $V$ is a vibrational operator. We do not give further explanations for the labels of these operators, since they will reduce to very simple forms in our calculations. It is sufficient to say that the $\Gamma$ 's are symmetry labels. These operators are coupled together (in the $T_{d}$ group) and the surrounding [...] stands for a symmetrized tensorial product, since the direction cosine and the rovibrational operator do not commute in general. This way, matrix elements are obtained through standard tetrahedral formalism techniques similar to the ones used for the dipole moment and the polarizability tensor of methane. More details are also given in Ref. [56]. As an example, we applied this general formalism to derive vibrationally induced quadrupole matrix elements that were subsequently used in the study of collision induced absorption in the $\nu_{2}$ band of methane $[66,67]$. The connection between the general operator of Eq. (8) and an angular operator related to methane in the interaction potential is made with

$$
\begin{aligned}
& \mathcal{D}^{\left(l_{1 g}, l_{1 g}, 0 \Gamma_{1}\right)} \equiv \\
& \equiv\left[\mathcal{D}^{\left(l_{1 g}, l_{1 g}, 0 \Gamma_{1}\right)} \times\left(\operatorname{Id}_{r}^{\left(A_{1}\right)} \times \operatorname{Id}_{v}^{\left(A_{1}\right)}\right)^{\left(A_{1}\right)}\right]^{\left(\Gamma_{1}\right)}
\end{aligned}
$$

that involves two identity operators related to rotation and vibration of methane. This is reminiscent of the fact that, for example, methane octopolar and hexadecapolar terms of the electrostatic interaction are permanent quantities, i.e. not rovibrationally induced ones.

Similarly, the (P.S.M.E.) of Eq. (4) are Perturber (nitrogen) Squared Matrix Elements,

$$
\begin{aligned}
& \text { (P.S.M.E. })^{\left(l_{2}\right)}\left(2,2^{\prime}\right)=V\left(\begin{array}{ccc}
J_{2} & l_{2} & J_{2^{\prime}} \\
0 & 0 & 0
\end{array}\right)^{2} \times \\
& \times \frac{\left|\left\langle J_{2} ; J_{2}\left\|\mathcal{D}^{\left(l_{2}, l_{2}\right)}\right\| J_{2^{\prime}} ; J_{2^{\prime}}\right\rangle\right|^{2}}{\left[J_{2}\right]\left[J_{2^{\prime}}\right]}
\end{aligned}
$$


The $V$ in this equation is a coupling symbol and is equal to the corresponding $3 J$ symbol, up to a phase factor. The notations for the matrix elements are analogous to those of Eq. (7), except that there is no need to define symmetrized quantities for homonuclear diatomic molecules like nitrogen.

The theory described above is consistent with the usual formalism of Robert and Bonamy, as applied to many molecular couples since decades. Recently, a possible mistake in the original derivation, regarding cumulant expansions $[68,69]$ for the Liouville states of the perturbers and the average over the populations of these states, has been reported [70-72]. Technically, Eq. (2) might need to be modified so that the average over the populations of the perturber states be placed inside the exponential, thus directly averaging $S_{2, f i,\{2\}}\left(r_{c}, v\right)$, and not outside. We have examined that point to some extent, and think that one should not confuse two things. The average over the populations of the perturber states is the first one. The matrix elements of the scattering operator over the perturber states, in the Liouville space, which is an averaging operation, is the second one. We believe that only the second is subject to re-exponentiation in Eq. (2), thus that the original derivation is correct. In fact, before obtaining that equation, the average over the "bath of perturbers", which consists in three operations (averages over the impact parameters, the relative velocities and the populations of the quantum states of the perturbers) has already been the subject of a cumulant expansion. This expansion allows to switch from the evolution operator related to the autocorrelation function of the dipole moment to an exponential form containing combined integrals of the interaction potential. This exponential form is essential, since its Fourier transform leads precisely to the Lorentzian profile for the considered line. Nonetheless, we do not exclude to do wrong in still relying to the original derivation of the theory. Practically, we tested this point by adding a special feature to our program generator (see section 2.3.2) so that it implements the modification described above. We did a comparison of the line broadening calculations in the $Q$ branch of the $\nu_{3}$ band of methane and found that the two cases differ by a few percent on average. This may come from the fact that the development of the scattering operator inside the exponential is truncated to order two. Anyway, such numerical comparisons do not constitute any mathematical proof. In addition, the modification of the theory in Ref. [71] seems to mean that, at fixed impact parameter and relative velocity, active molecules collide with perturbers that are not in definite quantum states, since this information is averaged out through the population factors placed inside the exponential 
terms of Eq. (2). According to these arguments, we would say that the three averaging operations that we collectively call the average over the "bath of perturbers" should be kept together. This conclusion may not be definitive, and we leave this point for the moment.

\subsection{Implementation}

In practice, the above theory happened to be very complicated to implement without the help of some automated procedures. We devote this section to these technical aspects since they represented an important part of the development of the theory. So, we have set up a modular system that allows to develop all the analytical calculations. We have used the MuPAD [73] symbolic computation software for such calculations. We also performed almost all the programming tasks through automated source code generation. For this, we used the PERL scripting language, the standard library [74] as well as additional packages available from the CPAN [75].

\subsubsection{Symbolic computation}

The set of symbolic computation codes takes, as arguments, a keyword for the molecular couple, the leading exponents for the short range repulsive and attractive interactions (12 and 6 , in our case, but any other values may be given), the maximum values of $l_{1}, l_{2}$ and $q$, the names of the point symmetry groups for the molecules (or none, if not applicable), the name of the relative trajectory model for the molecules (only the parabolic model is implemented, up to now).

With these settings, several tasks are performed. The set of coordinates for the equilibrium configurations of each molecule is defined (some generic molecules have been implemented). Then, the short range atom-atom interaction is calculated from two centers expansions of the inverse powers of the distance between the atoms in the two molecules. Point symmetry groups for the two molecules are accounted for and the potential is transformed to a symmetrized version. For us, $T_{d}$ is defined for methane and the relevant potential labels and terms are modified accordingly, and nothing is done for nitrogen. If defined, additional contributions are joined to the potential. For our case, we added two electrostatic contributions, as explained in section 2.2. Once done, the potential labels are read back and all the Fourier transforms of Eq. (5) are calculated analytically. The functions with fixed values of $l_{1}, l_{2}, m_{1}, m_{2}$ and a fixed power of the inverse radial coordinate are stored in files which names are generated using these labels and the keyword for the 
molecular couple. Also, when $l_{1} \neq 0$ and $l_{2} \neq 0$, the code is able to recognize if the numerical coefficients of the functions with $m \neq 0$ are proportional to the one of the function with the same labels, but $m=0$. In such a case, it merges the functions together accounting for the relevant numerical factors. Fortunatly, this is always the case for our molecular couple, which limits the amount of functions to calculate. Nevertheless, the number of functions can be quite large, depending on the settings for the orders of developments. For example, the calculations discussed below were done with $l_{1}+l_{2} \leq 6$ and $q_{\max }=14$, which corresponds to 845 generated functions.

All this is done only once with large values for the orders of developments. This way, several version of the source codes for actual calculations, with different settings, may be created.

\subsubsection{Source code generation}

To handle the large amount of calculations generated by the symbolic computation codes, we have developed a program generator written in PERL that produce FORTRAN 90/95 source codes for actual line broadening calculations. It makes use of PERL packages such as IO: :File that supplies object methods for filehandles, and Text: :Template that expands template text with embedded PERL code. For our needs, only a reduced set of 17 template files were needed [56]. These template files allowed us to build programs that create themselves their own source codes. The arguments of the generator include a keyword for the molecular couple, the maximum values of $l_{1}, l_{2}$ and $q$ (which may differ from the ones given during symbolic computation) and the name of the file containing the expressions of the coefficients of the potential. The keyword for the molecular couple is used, for example, to locate the files written by the MuPAD codes, but also to define non-ambigous file names for the generated source codes (in view of possible extension to some other molecular couple). For what concerns the Fourier transforms, the generator builds two kinds of procedures that perform some parts of the calculations of Eq. (5).

XY4A2_11_12_m1_m2.f90: This kind of procedure implements a calculation at fixed values of $l_{1}, l_{2}, m_{1}$ and $m_{2}$. As explained at the end of section 2.3.1, the sum over $m$ in Eq. (5) was done formerly. Only the sum other the inverse powers of the radial coordinate is done here. In practice, procedure subfunctions are created for all possible powers and the relevant combinations of these subfunctions with the numerical factors of 
the potential are built. Using the contains construct of the FORTRAN 90/95 syntax, the subfunctions are stored inside the calling procedure, which allows to limit the amount of generated files. Accounting for the fact that some functions are the same when projection numbers change in sign, we are left with 76 source files, corresponding to $l_{1}+l_{2} \leq 6$.

XY4A2_11_12.f90: This kind of procedure contains the calling sequence for the above procedures. Thus, at fixed values of $l_{1}$ and $l_{2}$, it implements the sum over $m_{1}$ and $m_{2}$ in Eq. (5). There are height such procedures, owing to the possible combinations of $l_{1}$ and $l_{2}$.

While doing this, the relevant numerical factors of the potential are numbered and accumulated in a dedicated procedure. Also, all the interface blocks (procedure prototypes) for the Fourier transforms procedures described above are generated and stored on the fly in separate modules. Then, the main program is generated and additional procedures for doing initializations, calculating dynamical factors, reading methane multipolar matrix elements, ... are also created.

Note that we have chosen to implement the calculations of multipolar matrix elements for methane independently from line broadening calculations. This was done by extending the set of codes dedicated to dipole moment which is part of the STDS package developed by the Dijon group [23, 76].

Note also that the program generator described above is not fully modular. We were principally concerned by the methane-nitrogen molecular couple. More generally, such procedure may be applied to any $\mathrm{XY}_{4}-\mathrm{A}_{2}$ generic couple. This would allow to address the cases of oxygen and hydrogen as perturbers, which we plan to do. For extensions to other molecular species, such as $\mathrm{CH}_{3} \mathrm{D}$, we'll need to account for the multiplicity indices for $l_{1}$ and $l_{2}$, as explained in section 2.2, after Eq. (7). Thus, for example, the file naming scheme for source codes described above will need to be extended, with the use of adapted PERL regular expressions constructs. In fact, developing the theory for $\mathrm{CH}_{3} \mathrm{D}$ comes along with doing so for $\mathrm{CH}_{4}$ and should be one of the next step of the present work.

\subsection{Numerical data}

Wavefunctions and energies for methane are calculated using the latest version of the STDS computer package, consistent with the most up to date line positions and intensities analysis [23]. For a given experimental data set, 
line assignments are read and only the relevant multipolar matrix elements and energy spacings are calculated.

For nitrogen, we used a simple model where the rotational energies are given by

$$
E_{\mathrm{N}_{2}}\left(J_{2}\right) / h c=B_{0} J_{2}\left(J_{2}+1\right)-D_{0} J_{2}^{2}\left(J_{2}+1\right)^{2}
$$

where $h$ is Planck's constant. We took $B_{0}=1.98956 \mathrm{~cm}^{-1}[77,78]$ (average value from these references) and $D_{0}=5.763 \times 10^{-6} \mathrm{~cm}^{-1}$ [77]. Since we did calculations at room temperature, we did not account for vibrational energies. We calculate the population factor for perturbers using

$$
\begin{aligned}
& \rho_{\{2\}} \equiv \rho\left(J_{2}\right)=\frac{S_{\mathrm{N}_{2}}\left(J_{2}\right)\left(2 J_{2}+1\right)}{Z_{\mathrm{R}, \mathrm{N}_{2}}(T)} \times \\
& \quad \times \exp \left(-E_{\mathrm{N}_{2}}\left(J_{2}\right) / K_{B} T\right)
\end{aligned}
$$

This expression corresponds to the rigid rotor approximation, which is the main contribution to the rotational energies. The $S_{\mathrm{N}_{2}}\left(J_{2}\right)$ are the rotational statistical weights,

$$
S_{\mathrm{N}_{2}}\left(J_{2}\right)=\alpha_{\mathrm{N}_{2}}+\beta_{\mathrm{N}_{2}}(-1)^{J_{2}}
$$

with $\alpha_{\mathrm{N}_{2}}=3 / 2$ and $\beta_{\mathrm{N}_{2}}=1 / 2$. The rotational partition function, $Z_{\mathrm{R}_{\mathrm{N}} \mathrm{N}_{2}}(T)$, is calculated by summing the un-normalized populations with a definite cutoff for $J_{2}$, depending on the value of the population at the peek of the distribution. For example, at room temperature, the peak is found at $J_{2}=7$ and the cut-off is determined to be $J_{2}=34$.

For what concerns the interaction potential, we have adopted the parameters from Ref. [44] or average values from Refs. [44, 79] (for the electrostatic terms). All the values below were taken as is, i.e. without any attempt to adjust them to achieve better comparison to the experimental data. The multipolar moments are $\Omega_{(1), \mathrm{CH}_{4}}=2.45$ D. $\AA^{2}, \Phi_{(1), \mathrm{CH}_{4}}=4.725 \mathrm{D} . \AA^{3}$ and $\Theta_{(2), \mathrm{N}_{2}}$ $=-1.375 \mathrm{D} . \AA$. We write the short range interaction between atom $1 i$ of methane and atom $2 j$ of nitrogen as

$$
V_{1 i, 2 j}\left(r_{1 i, 2 j}\right)=\frac{d_{1 i, 2 j}}{r_{1 i, 2 j}^{12}}-\frac{e_{1 i, 2 j}}{r_{1 i, 2 j}^{6}}
$$

The parameters are $d_{C N}=0.512 \times 10^{-14} \mathrm{~J} . \AA^{12}, d_{H N}=0.325 \times 10^{-15} \mathrm{~J} . \AA^{12}$,

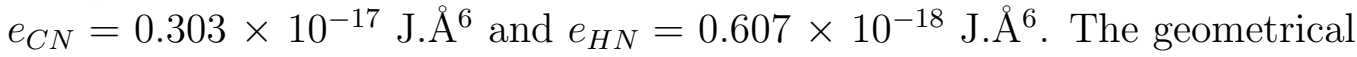
parameters are the equilibrium bond lengths, or half of it for nitrogen, $r_{C H}=$ $1.086 \AA$ and $r_{G N}=r_{N N} / 2=0.549 \AA$. See also Ref. [56] for more details about these points. 


\section{Applications}

To test the consistency and overall accuracy of the theory of section 2.2, we did calculations for lines in the $\nu_{4}$ band, the $\nu_{3}$ band and the octad spectral region of methane. The experimental values for the line positions and line broadening coefficients were taken from Refs. [34, 42, 47, 52, 80]. For the $\nu_{4}$ band and the octad region, the perturber was in fact air [34, 42], for which nitrogen contributes to about $80 \%$. Also, according to Fig. 7 of Ref. [80], Table 3 of Ref. [52] ( $\nu_{3}$ band $Q$ branch) and to Refs. [39] ( $\nu_{4}$ band broadened by $\mathrm{N}_{2}$ ) and [41] ( $\nu_{4}$ band broadened by $\left.\mathrm{O}_{2}\right)$, we expected the ratio of $\mathrm{N}_{2}$-broadening to air-broadening for a given line to be near unity, on average. Obviously, we will need to revise that crude approximation by extending our calculations to the case of oxygen as the perturber for better consistency check. In fact, in the present work, we did not expect to reach very good quantitative agreement, in the sense that the majority of the calculations would fall inside the uncertainty intervals of the corresponding measurements. For a non polar system like methane-notrogen, this ideal situation can't clearly be reached using a semiclassical model. Thus, we considered that to compare air measurements to calculations with nitrogen as the perturber would suffice for the moment. All the measurements were done at room temperature.

The calculations were all done with $l_{1}+l_{2} \leq 6$, for what concerns the angular operators of the potential. Regarding the associated radial functions, several tests of convergence were made. We have finally chosen to show the results with $q_{\max }=14$ (845 Fourier transforms). As one can expect, the average amount of matrix elements for each calculated line grows with the polyad number. It ranges from some hundreds in $\nu_{4}$ to about 2000 in $\nu_{3}$ and to about 7000 in the octad region. We will discuss the results from various plots in the next sections. The associated full tabulated results (text files) are provided in the supplementary material of this work.

\subsection{The $\nu_{4}$ band}

The 446 measurements range from $1159.3 \mathrm{~cm}^{-1}$ to $1494.7 \mathrm{~cm}^{-1}$ with angular momenta up to $J^{\prime \prime}=20$ [42]. From these data, we found that one line at wavenumber $1267.21035 \mathrm{~cm}^{-1}$ had an upper level order index that was too large, i.e. larger that the size of the corresponding Hamiltonian block. We disregarded it, since it made our calculations abort. We also found that three other lines were misassigned. We left them as is in the calculations. The lines 
in question are located at wavenumbers $1219.64879 \mathrm{~cm}^{-1}, 1221.87989 \mathrm{~cm}^{-1}$ and $1223.35630 \mathrm{~cm}^{-1}$ and seem to be some $\mathrm{P}(12)$ and/or $\mathrm{P}(13)$ lines.

\section{[Figs. 1, 2 and Table 1 go near here]}

Measurements and calculations are shown on Fig. 1 where we have used different symbols for the types of symmetries of the lines. Note that for this figure, we only kept the data for which the observed minus calculated line broadening coefficients do not exceed $\pm 50 \%$. Thus, 19 lines were disregarded and 426 lines are shown on Fig. 1. The histogram of the differences between observed and calculated line broadening coefficients is shown on Fig. 2. Details for the 19 disregarded lines are given in Table 1. For the 426 other calculated lines, see the corresponding data file with tabulated results in the supplementary material. It is seen from Table 1 that all the lines have rather large values of $J$ and that the fits of experimental spectra made in Ref. [42] lead to very small values for the line broadening coefficients, compared to the calculated ones. According to table 3 of Ref. [42], none of these lines were fitted with the inclusion of line-mixing. Presumably, for these high $J$ values, intra-manifold, as well as inter-manifold, line-mixing should have been accounted for. As a consequence of strong correlations between the parameters defining the line shapes [52], the fitted values for these lines may be seen as effective. If the line broadening coefficients were found to be so small, then this means that the dispersive contributions to the line shapes are possibly important. For this reason, we will not recommand to use the experimental nor the calculated line broadening coefficients of Table 1 as is, i.e. for isolated line shapes calculations. Anyway, at room temperature, the intensities of these lines are rather small.

As may be seen from Fig. 1, for 426 lines out of 446 in the $\nu_{4}$ band, the calculations compare reasonably well to the measurements, although these latter ones were done with air as the perturber. We see also that at fixed $J$ or, equivalently, at fixed wavenumber, the dispersion of the calculated data is less important than that of the experimental ones. We found the same deficiency of the model in the case of methane perturbed by argon and helium $[62,63]$. Nevertheless, we see from Fig. 2 that the differences are within $\pm 15 \%$ for most of the lines, which provides some confidence regarding our model. We notice also that, although the histogram exhibits a bell shape, globally, centered around $0 \%$, this shape is not very regular. Thus, differences between measurements and calculations are either too large 
or too small depending on the lines, as is clear also from Fig. 1. Before investigating and discussing further that point, we would need first to do proper air broadening calculations.

\subsection{The $\nu_{3}$ band}

The 182 measurements range from $2916.2 \mathrm{~cm}^{-1}$ to $3122.8 \mathrm{~cm}^{-1}$ with angular momenta up to $J^{\prime \prime}=13[47,52,80]$. Measurements for the $\nu_{3}$ band Q branch [80] were re-examined to include line-mixing, Dicke narrowing and speed dependent line shapes in the analysis [52]. Such refinments allowed to improve and extend the fittings of the recorded spectra. Thus, we did calculations for the most recent line shape parameters for that spectral region.

\section{[Figs. 3, 4 and 5 go near here]}

Measurements and calculations are shown on Figs. 3 and 4 and the histogram of the associated differences is shown on Fig. 5. We found that one line located at $3095.13038 \mathrm{~cm}^{-1}$, assigned to $\mathrm{R}(7), F_{2}, 2,29$, was in fact misassigned. The central part of Fig. 4 allows to see an expanded view of the data for the $\nu_{3}$ band $\mathrm{Q}$ branch. Details for the $J$ manifolds in the $\mathrm{P}$ and $\mathrm{R}$ branches are seen on the left and the right of this figure, respectively. As seen from Figs. 3 and 4, the calculations compare fairly well to the measurements. We found again that, at fixed wavenumber, the scatter of calculated data is underestimated. The histogram of Fig. 5 shows that most of the differences are between $-10 \%$ and $+20 \%$. Different factors may explain that $\approx 5 \%$ positive mean difference. The spectra analysis of Refs. [47, 52] showed that the Voigt line shape was inadapted, and that refined models had to be used. The use of different refinments, for example the Rautian [81] or Galatry [82] line shape models, may lead to fits of the same overall qualities, but with individual line broadening coefficients differing by a few percents $[39,41]$. Obviously, the intrinsic defaults of the model are other factors that explain these results. We will discuss some common points, i.e. that apply to all data sets, regarding the model in section 4 . Line mixing was accounted for to fit the spectra of the $\nu_{3}$ band. By inspecting the top panel of Fig. 4, we see that some $E$ type lines in the Q branch were found to have quite low values for the line broadening coefficients, relative to the calculations. On the contrary, for one $F$ type doublet in the $\mathrm{R}$ branch, the values are quite large. In Refs. [47, 52], for the line mixing parameters, themretical values deduced from semiclassical calculations were used as constraints or initial values for 
the fits. We note that the highest discrepancies (Fig. 4) do not exceed 50\%, absolute value. We observe that, for the $\nu_{4}$ and $\nu_{3}$ bands, large deficiencies happen mostly for $E$ type lines, but we see no explanation for this. From Fig. 4, we see that the relative ordering of the measurements with respect to the symmetry labels of the lines (which reflect the fine structure of the energy levels involved) is globally well accounted for by the calculations. We also compared the present calculations to those of Ref. [44] for 145 data out of 182 . Line by line details are provided in the supplementary material. We found that the differences relative to our calculations range between $\approx-2 \%$ and $\approx-12 \%$ with a mean value of $\approx-7 \%$. We conclude that the lack of the imaginary part of the $S_{2}$ function in Eq. (2) may play a role in the small positive mean difference between our calculations and the measurements.

\subsection{The octad region}

The 1013 measurements range from $4101.8 \mathrm{~cm}^{-1}$ to $4627.8 \mathrm{~cm}^{-1}$ with angular momenta up to $J^{\prime \prime}=16$ [34]. 750 lines were calculated, which was thought to be sufficient for a qualitative discussion of the results.

\section{[Figs. 6 and 7 go near here]}

Measurements and calculations are shown on Fig. 6 and the histogram of the associated differences is shown on Fig. 7. One line located at 4265.42181 $\mathrm{cm}^{-1}$, assigned to $\mathrm{P}(5), F_{2}, 1,33$, is not shown on these figures because the value of the broadening coefficient is $0.0904 \mathrm{~cm}^{-1} \cdot \mathrm{atm}^{-1}$. We think that this is a typo in the experimental data file. This octad spectral region consists mainly in two vibrational bands. The $\nu_{1}+\nu_{4}$ band is around $4223 \mathrm{~cm}^{-1}$ and the $\nu_{3}+\nu_{4}$ band is around $4319 \mathrm{~cm}^{-1}$ [34]. The latter has a greater vibrational degeneracy, which is reflected by the trends in the line broadening values on Fig. 6. We see from this figure that the global arrangement of data that results from the presence of vibrational subbands is well accounted for by the calculations. Again, the spread of data at fixed wavenumber is always underestimated by the calculations. We see also in the bottom panel of Fig. 6 that the differences show less scattering than in the case of $\nu_{4}$ (Fig. 1). The fits in the octad region were done with the standard Voigt line shape [34] and we found (Fig. 7) that the differences range from $-47 \%$ to $+25 \%$ for the 750 calculated lines. For most of these lines, the differences are between $-10 \%$ and $+20 \%$. Thus, the results are of the same overall quality as those for the $\nu_{4}$ and $\nu_{3}$ bands. Also, the histogram of Fig. 7 is seen to be centered around 
$+8 \%$. While testing the convergence of the development of the potential, we found that the calculations in that spectral region made with $q_{\max }=10$ and $q_{\max }=14$ differ by about $4 \%$ on average. Thus, increasing further the value of $q_{\max }$ will certainly not provide better calculations. One may also invoke the lack of the imaginary part of the $S_{2}$ function to explain these results. Obviously, we'll need also to do proper air broadening calculations before any further discussion. As for $\nu_{4}$ and $\nu_{3}$, the shape of the histogram shows that some measurements are either too large or too small relative to calculations.

\section{Discussion}

\subsection{The interaction potential}

This is the first point to address in the theory. We found that the contribution from the electrostatic part of the potential in the calculations was about $1 \%$ or less. Thus, any refinements in the corresponding parameters will not help. We were puzzled by this result that seemed inconsistent with earlier calculations [83-88] that did not include any short range interactions. Possibly, the different theory used, that include a cut-off procedure for the interruption function, may explain in part this result. Obviously, we carefully checked the units, conversion factors and orders of magnitude of the various quantities in our calculations but did not find any incoherence. We then did some numerical tests using the experimental data recorded in the $\nu_{3}$ band Q branch of methane. We found that the calculated line broadening coefficients with only the electrostatic terms, and the ones with only the short range terms limited to $l_{1}=3, l_{2}=0$ or 2 and $q=3$ or 5 (lowest order terms) are of the same order of magnitude (about 1\% of the experimental data). We then did other calculations with only the short range terms limited to $\left\{l_{1}=3, l_{2}=0, q=3,5,7,9,11,13\right\}$, and $\left\{l_{1}=3, l_{2}=2, q=5,7,9\right.$, $11,13\}$. This calculated set was found to contribute for about $40 \%$ to $60 \%$ to the experimental data. The fine structure dependencies of the data were also ssen to be quite consistent with the ones of experimental data.

We did several other tests on the full short range (atom-atom) interaction. For this, and the calculations described above, we defined a special option to our program generator so that to read the labels of the radial functions that we want to include in the calculations in a separate file. This way, appropriate Fourier transforms files are selected and the program is build accordingly. 
We found that increasing the order of development for radial functions provides some sort of convergence, in that histograms of differences happened to be more likely centered around $0 \%$. In reality, we artificially add contributions to the interruption functions that all get almost the same values at fixed values of the rotational quantum numbers. As a consequence, the energy fine structure dependences of the calculated line broadening coefficients is averaged out to some extent and is always found to be underestimated. Although this allows to reproduce in part these fine structure dependencies of the broadening parameters, this is a great defficiency of the model. It would perhaps be more appropriate to limit the calculations to lower orders and to determine a better set of atom-atom parameters, possibly dependent on the radial functions. We shall test this assumption by using a recently reported ab initio methane nitrogen potential [89]. Alternatively, other functional forms for the potential radial functions could be tested.

\subsection{Collision dynamics}

The classical nature of the relative trajectories of the molecules may also be discussed. These trajectories are driven by the isotropic interaction, which implies a simplified conservation rule for the orbital angular momentum. Furthermore, we used a parabolic approximation for the time dependences of the trajectories near the points of closest approach. Possibly, the use of exact trajectories, i.e. the solutions of the Hamilton's equations using the isotropic potential, may ameliorate the calculations. This is a point that we wish to address, after the one concerning the radial functions of the methanenitrogen potential (section 4.1).

Also, implicitly, we approximate each molecule by a sphere. As discussed above, the leading broadening mechanisms for methane perturbed by nitrogen are short range collisions, at room temperature. In such conditions, it appears necessary to account for the anisotropy of the molecules in the treatment of collision dynamics. To our knowledge, theories that include such refinments were developed for simple molecular systems, and in any case not for non-linear molecules like methane as the absorber. Anyway, it seems that practical calculations are only tractable for linear molecules up to now [90-94]. Further investigations on these points are obviously needed.

\subsection{Temperature dependencies}

We did comparisons of our calculations to measurements made at room temperature. We will need, depending on the application, to consider other 
physical conditions. In particular, realistic predictions of the nitrogen line widths for methane lines at temperatures around $100 \mathrm{~K}$ or below are missing. This is true for the spectral regions that we considered in this work, as well as for the next polyads with higher wavenumbers (tetradecad, icosad, ... ). We expect our semiclassical model to not be adapted to such low temperatures. One reason is that less quantum states of the molecules are significantly populated. Also, the various energies involved in the collision dynamics are lower, so that a classical treatment of it is less appropriate.

As a first attempt, one may predict the temperature dependences of the line widths using a power law,

$$
\gamma_{f i}(T)=\gamma_{f i}\left(T_{0}\right)\left[\frac{T_{0}}{T}\right]^{n_{f i}}
$$

where $n_{f i}$ is the temperature exponent for the considered line, and $T_{0}$ is a reference temperature. usually the ambient one. This law has been widely used and is often stated as empirical, although it has some theoretical justification $[95,96]$ for a simplified potential model. Also, the hard sphere model predicts a constant value of 0.5 , whatever the spectral line. Temperature exponents have been measured for methane, e.g. in the $\nu_{4}$ band [42], and these ones were found to be quite transition specific. As explained above, improvements for our methane line broadening theory have to be done. Thus, investigations on the temperature dependences of line widths are left to future works.

\section{Conclusion and perspectives}

Semiclassical line broadening coefficients calculations for methane perturbed by nitrogen have been done. For this, a symmetry adapted formalism was developed. This one allows to use directly the eigenfunctions and energies as obtained from accurate polyads analysis of methane. We developed some automated procedures for the practical implementation of the theory. The model is free of any adjusted parameter, which makes it somewhat complicated.

We compared our calculations to measurements made in the first three polyads of methane, at room temperature, with air and nitrogen as the perturbers. We found good overall qualitative agreement at worst, and good quantitative agreement at best. When line-mixing was possibly involved in the experimental spectra, we've seen that discrepencies can be (very) large 
between measurements and calculations for some lines. For such large differences, deficiencies of the model are also present, and the reliability of either parameters is questionnable. For the air measurements, we will need to extend our model to the case of oxygen as the perturber before any further investigation. We found that, in general, at room temperature, line broadening is essentially due to the short range interactions between the molecules, as expected for such non-polar molecular species.

The methane nitrogen interaction potential that we have used does not contain any vibrational contribution. Due to the complexity of the problem, we did not include the imaginary parts of the $S_{2}$ function of Eq. (2). Symbolic computation might help in part for these points. Thus, line shifts could not be calculated in the present work. In fact, we expect also vibrational effects to play a role for the line widths in the case of perturbation allowed vibrational bands like $\nu_{2}$ [97]. This is also possibly the case for higher overtone and combination vibrational bands. Finally, we did not address the question of the temperature dependences of the collisional parameters, nor that of the modeling of line-mixing between lines. These important points should be the subject of future works.

\section{Acknowledgments}

This work is part of the French ANR Project "CH4@Titan" [98]. We acknowledge financial support from the LEFE-CHAT French National Program. We also thank the SpecMo GdR number 3152 of the CNRS [99] and the "Pôle de Sciences Planétaires Bourgogne Franche-Comté" [100].

\section{Supplementary material}

Ref. [56] and data files are provided with the online version of this article at ScienceDirect (www.science-direct.com), or at ... 


\section{References}

[1] Boudon V, Champion JP, Gabard T, Pierre G, Loëte M, Wenger C. Spectroscopic tools for remote sensing of greenhouse gases $\mathrm{CH}_{4}, \mathrm{CF}_{4}$ and $\mathrm{SF}_{6}$. Environ Chem Lett 2003;1:86-91.

[2] Khalil MA. Non- $\mathrm{CO}_{2}$ greenhouse gases in the atmosphere. Annu Rev Energy Environ 1999;24:645-661.

[3] Formisano V, Atreya S, Encrenaz T, Ignatiev N, Giuranna M. Detection of methane in the atmosphere of Mars. Science 2004;306:17581761.

[4] Caradini A, Filacchione G, Capaccioni F, Cerroni P, Adriani A, Brown RH, et al. CASSINI/VIMS-V at Jupiter: Radiometric test and data results. Planetary and Space Science 2004;52:661-670.

[5] Irwin PGJ, Shira K, Bowles N, Taylor FW, Calcutt SB. Methane absorption in the atmosphere of Jupiter from 1800 to $9500 \mathrm{~cm}^{-1}$ and implications for vertical cloud structure. Icarus 2005;176:255-271.

[6] Tran H, Flaud PM, Fouchet T, Gabard T, Hartmann JM. Model, software and database for line-mixing effects in the $\nu_{3}$ and $\nu_{4}$ bands of $\mathrm{CH}_{4}$ and tests using laboratory and planetary measurements. II. $\mathrm{H}_{2}$ (and He) broadening and the atmosphere of Jupiter and Saturn. J Quant Spectrosc Radiat Transfer 2006;101:306-324.

[7] Coustenis A, Salama A, Schulz B, Lellouch E, Encrenaz T, Ott S, et al. Past and future space observations of Titan in the infrared and submm ranges: ISO, Cassini and FIRST. In: Pilbratt GL, Cernicharo J, Heras AM, Prusti T, Harris R, editors. The promise of the Herschel Space Observatory, 12-15 December 2000, Toledo, Spain. vol. SP-460. ESA; 2001.

[8] Griffith CA, Penteado P, Rannou P, Brown R, Boudon V, Baines $\mathrm{KH}$, et al. Evidence for a polar ethane cloud on Titan. Science 2006;313:1620-1622.

[9] Negrão A, Coustenis A, Lellouch E, Maillard JP, Rannou P, Schmitt $\mathrm{B}$, et al. Titan's surface albedo variations over a Titan season 
from near-infrered CFHT/FTS spectra. Planetary and Space Science 2006;54:1225-1246.

[10] Coustenis A, Negrão A, Salama A, Schulz B, Lellouch E, Rannou P, et al. Titan's 3-micron spectral region from ISO high-resolution spectroscopy. Icarus 2006;180:176-185.

[11] Negrão A, Hirtzig M, Coustenis A, Gendron E, Drossart P, Rannou P, et al. The $2-\mu \mathrm{m}$ spectroscopy of Huygens probe landing site on Titan with Very Large Telescope/Nasmyth Adaptive Optics System NearInfrared Imager and Spectrograph. J Geophys Res 2007;112:E02S92.

[12] Sromovsky LA, Fry PM. The methane abundance and structure of Uranus' cloud bands inferred from spatially resolved 2006 Keck grism spectra. Icarus 2008;193:252-266.

[13] Orton GS, Encrenaz T, Leyrat C, Puetter R, Friedson AJ. Evidence for methane escape and strong seasonal and dynamical perturbations of Neptune's atmospheric temparatures. Astron Astrophys 2007;473:L5L8.

[14] Stansberry JA, Spencer JR, Schmitt B, Benchkoura AI, Yelle RV, Lunine JI. A model for the overabundance of methane in the atmospheres of Pluto and Triton. Planetary and Space Science 1996;44(9):10511063.

[15] Lellouch E, Sicardy B, de Bergh C, Käufl HU, Kassi S, Campargue A. Pluto's lower atmosphere structure and methane abundance from high-resolution spectroscopy and stellar occultations. Astronomy and Astrophysics 2009;495:L17-L21.

[16] Swain MR, Vasisht G, Tinetti G. The presence of methane in the atmosphere of an extrasolar planet. Nature 2008;452:329-331.

[17] Swain MR, Tinetti G, Vasisht G, Deroo P, Griffith C, Bouwman J, et al. Water, Methane, and Carbon Dioxide Present in the Dayside Spectrum of the Exoplanet HD 209458b. Astrophys J 2009;704:1616-1621.

[18] Villanueva GL, Mumma MJ, Novak RE. Strong release of methane on Mars: Evidence of biology or geology? Geochim Cosmochim Acta 2009;73:A1384-A1384. 
[19] Lefèvre F, Forget F. Observed variations of methane on Mars unexplained by known atmospheric chemistry and physics. Nature 2009;460:720-723.

[20] Jennings DE, Romani PN, Bjoraker GL, Sada PV, Nixon CA, Lunsford $\mathrm{AW}$, et al. ${ }^{12} \mathrm{C} /{ }^{13} \mathrm{C}$ Ratio in Ethane on Titan and Implications for Methane's Replenishment. J Phys Chem A 2009;113:11101-11106.

[21] Suits AG. Titan: A Strangely Familiar World. J Phys Chem A 2009;113:11097-11098.

[22] Boudon V, Rey M, Loëte M. The vibrational levels of methane obtained from analyses of high-resolution spectra. J Quant Spectrosc Radiat Transfer 2006;98:394-404.

[23] Albert S, Bauerecker S, Boudon V, Brown LR, Champion JP, Loëte $\mathrm{M}$, et al. Global analysis of the high resolution infrared spectrum of methane ${ }^{12} \mathrm{CH}_{4}$ in the region from 0 to $4800 \mathrm{~cm}^{-1}$. Chem Phys 2009;356:131-146.

[24] Liu AW, Kassi S, Campargue A. High sensitivity CW-cavity ring down spectroscopy of $\mathrm{CH}_{4}$ in the $1.55 \mu \mathrm{m}$ transparency window. Chem Phys Lett 2007;447:16-20.

[25] Kassi S, Gao B, Romanini D, Campargue A. The near-infrared (1.30$1.70 \mu \mathrm{m})$ absorption spectrum of methane down to $77 \mathrm{~K}$. Phys Chem Chem Phys 2008;10:4410-4419.

[26] Sciamma-O'Brien E, Kassi S, Gao B, Campargue A. Experimental low energy values of $\mathrm{CH}_{4}$ transitions near $1.33 \mu \mathrm{m}$ by absorption spectroscopy at 81 K. J Quant Spectrosc Radiat Transfer 2009;110:951-963.

[27] Kassi S, Romanini D, Campargue A. Mode by Mode CW-CRDS at $80 \mathrm{~K}$ : Application to the $1.58 \mu \mathrm{m}$ transparency window of $\mathrm{CH}_{4}$. Chem Phys Lett 2009;477:17-21.

[28] Gao B, Kassi S, Campargue A. Empirical low energy values for methane transitions in the $5852-6181 \mathrm{~cm}^{-1}$ region by absorption spectroscopy at 81 K. J Mol Spectrosc 2009;253:55-63. 
[29] Hirtzig M, Tokano T, Rodriguez S, le Mouelic S, Sotin C. A review of Titan's atmospheric phenomena. Astron Astrophys Rev 2009;17:105147.

[30] Irwin PGJ, Sromovsky LA, Strong EK, Sihra K, Teanby NA, Bowles N, et al. Improved near-infrared methane band models and k-distribution parameters from 2000 to $9500 \mathrm{~cm}^{-1}$ and implications for interpretation of outer planet spectra. Icarus 2006;181:309-319.

[31] Lepère M, Valentin A, Henry A, Camy-Peyret C, Lengelé M, Populaire JC, et al. Diode laser spectroscopy: Temperature dependence of $R(0)$ line in the $\nu_{4}$ band of $\mathrm{CH}_{4}$ perturbed by $\mathrm{N}_{2}$ and $\mathrm{O}_{2}$. J Mol Spectrosc 2005;233:86-92.

[32] Mondelain D, Chelin P, Valentin A, Hurtmans D, Camy-Peyret C. Line profile study by diode laser spectroscopy in the ${ }^{12} \mathrm{CH}_{4} \nu_{2}+\nu_{4}$ band. J Mol Spectrosc 2005;233:23-31.

[33] Predoi-Cross A, Brown LR, Malathy Devi V, Brawley-Tremblay M, Chris Benner D. Multispectrum analysis of ${ }^{12} \mathrm{CH}_{4}$ from 4100 to 4635 $\mathrm{cm}^{-1}$ : 1. Self-broadening coefficients (widths and shifts). J Mol Spectrosc $2005 ; 232: 231-246$.

[34] Predoi-Cross A, Brawley-Tremblay M, Brown LR, Malathy Devi V, Chris Benner D. Multispectrum analysis of ${ }^{12} \mathrm{CH}_{4}$ from 4100 to 4635 $\mathrm{cm}^{-1}$ : II. Air-broadening coefficients (widths and shifts). J Mol Spectrosc 2006;236:201-215.

[35] Predoi-Cross A, Unni AV, Heung H, Malathy Devi V, Chris Benner D, Brown LR. Line mixing effects in the $\nu_{2}+\nu_{3}$ band of methane. J Mol Spectrosc 2007;246:65-76.

[36] Mondelain D, Payan S, Deng W, Camy-Peyret C, Hurtmans D, Mantz AW. Measurement of the temperature dependence of line mixing and pressure broadening parameters between 296 and $90 \mathrm{~K}$ in the $\nu_{3}$ band of ${ }^{12} \mathrm{CH}_{4}$ and their influence on atmospheric methane retrievals. J Mol Spectrosc 2007;244:130-137.

[37] Tumuhimbise AT, Hurtmans D, Mondelain D, Mantz AW. Nitrogenpressure shifts in the $\nu_{3}$ band of methane measured at several temperatures between 300 and 90 K. J Mol Spectrosc 2008;252:239-241. 
[38] Frankenberg C, Warneke T, Butz A, Aben I, Hase F, Spietz P, et al. Pressure broadening in the $2 \nu_{3}$ band of methane and its implication on atmospheric retrievals. Atmos Chem Phys 2008;8:5061-5075.

[39] Martin B, Lepère M. $\mathrm{N}_{2}$-broadening coefficients in the $\nu_{4}$ band of ${ }^{12} \mathrm{CH}_{4}$ at room temperature. J Mol Spectrosc 2008;250:70-74.

[40] Mondelain D, Camy-Peyret C, Deng W, Payan S, Mantz AW. Study of molecular line parameters down to very low temperature. Applied Physics B: Lasers and Optics 2008;90:227-233.

[41] Martin B, Lepère M. $\mathrm{O}_{2}$ - and air-broadening coefficients in the $\nu_{4}$ band of ${ }^{12} \mathrm{CH}_{4}$ at room temperature. J Mol Spectrosc 2009;255:6-12.

[42] Smith MAH, Benner DC, Predoi-Cross A, Devi VM. Multispectrum analysis of ${ }^{12} \mathrm{CH}_{4}$ in the $\nu_{4}$ band: I. Air-broadened half widths, pressure-induced shifts, temperature dependences and line mixing. J Quant Spectrosc Radiat Transfer 2009;110:639-653.

[43] Lyulin OM, Nikitin AV, Perevalov VI, Morino I, Yokota T, Kumazawa $\mathrm{R}$, et al. Measurements of $\mathrm{N}_{2^{-}}$and $\mathrm{O}_{2}$-broadening and shifting parameters of methane spectral lines in the $5550-6236 \mathrm{~cm}^{-1}$ region. J Quant Spectrosc Radiat Transfer 2009;110:654-668.

[44] Antony BK, Niles DL, Wroblewski SB, Humphrey CM, Gabard T, Gamache RR. $\mathrm{N}_{2^{-}}, \mathrm{O}_{2^{-}}$and air-broadened half-widths and line shifts for transitions in the $\nu_{3}$ band of methane in the 2726- to $3200-\mathrm{cm}^{-1}$ spectral region. J Mol Spectrosc 2008;251:268-281.

[45] Pieroni D, Nguyen-Van-Thanh, Brodbeck C, Claveau C, Valentin A, Hartmann JM, et al. Experimental and theoretical study of line mixing in methane spectra. I. The $\mathrm{N}_{2}$-broadened $\nu_{3}$ band at room temperature. J Chem Phys 1999;110:7717-7732.

[46] Pieroni D, Nguyen-Van-Thanh, Brodbeck C, Hartmann JM, Gabard T, Champion JP, et al. Experimental and theoretical study of line mixing in methane spectra. II. Influence of the collision partner (He and Ar) in the $\nu_{3}$ IR band. J Chem Phys 1999;111:6850-6863. 
[47] Pine AS, Gabard T. Speed-dependent broadening and line mixing in $\mathrm{CH}_{4}$ perturbed by Ar and $\mathrm{N}_{2}$ from multispectrum fits. J Quant Spectrosc Radiat Transfer 2000;66:69-92.

[48] Pieroni D, Hartmann JM, Chaussard F, Michaut X, Gabard T, SaintLoup R, et al. Experimental and theoretical study of line mixing in methane spectra. III. The Q branch of the Raman $\nu_{1}$ band. J Chem Phys 2000;112:1335-1343.

[49] Pieroni D, Nguyen-Van-Thanh, Brodbeck C, Hartmann JM, Gabard T, Champion JP, et al. Experimental and theoretical study of line mixing in methane spectra. IV. Influence of the temperature and of the band. J Chem Phys 2000;113:5776-5783.

[50] Grigoriev IM, Filippov NN, Tonkov MV, Gabard T, Le Doucen R. Estimation of line parameters under line mixing effects: the $\nu_{3}$ band of $\mathrm{CH}_{4}$ in helium. J Quant Spectrosc Radiat Transfer 2001;69:189-204.

[51] Grigoriev IM, Filippov NN, Tonkov MV, Champion JP, Gabard T, Le Doucen $\mathrm{R}$. Line parameters and shapes of high clusters: $\mathrm{R}$ branch of the $\nu_{3}$ band of $\mathrm{CH}_{4}$ in He mixtures. J Quant Spectrosc Radiat Transfer 2002;74:431-443.

[52] Pine AS, Gabard T. Multispectrum fits for line mixing in the $\nu_{3}$ band Q branch of methane. J Mol Spectrosc 2003;217:105-114.

[53] Tran H, Flaud PM, Gabard T, Hase F, von Clarmann T, Camy-Peyret $\mathrm{C}$, et al. Model, software and database for line-mixing effects in the $\nu_{3}$ and $\nu_{4}$ bands of $\mathrm{CH}_{4}$ and tests using laboratory and planetary measurements. I. $\mathrm{N}_{2}$ (and air) broadenings and the Earth atmosphere. J Quant Spectrosc Radiat Transfer 2006;101:284-305.

[54] Champion JP, Loëte M, Pierre G. Spherical Top Spectra. In: Narahari Rao K, Weber A, editors. Spectroscopy of the earth's atmosphere and interstellar medium. Academic Press Inc. U.S.A.; 1992. p. 339-422.

[55] Boudon V, Champion JP, Gabard T, Loëte M, Michelot F, Pierre G, et al. Symmetry-adapted tensorial formalism to model rovibrational and rovibronic spectra of molecules pertaining to various point groups. J Mol Spectrosc 2004;228:620-634. 
[56] Gabard T. The $\mathrm{XY}_{4}-\mathrm{A}_{2}$ system : Potential, Line Broadening Theory, Resonance Functions, Symbolic Computation and Source Code Generation. Internal report. Laboratoire Interdisciplinaire Carnot de Bourgogne (ICB), UMR 5209 CNRS; 2009.

[57] Hartmann JM, Boulet C, Robert D. Collisional Effects on Molecular Spectra. Laboratory Experiments and Models, Consequences for Applications. Elsevier B.V., Amsterdam, The Netherlands; 2008.

[58] Robert D, Bonamy J. Short range force effects in semiclassical molecular line broadening calculations. J Phys (Paris) 1979;40:923-943.

[59] Antony BK, Gamache RR. Self-broadened half-widths and self-induced line shifts for water vapor transitions in the $3.2-17.76 \mu \mathrm{m}$ spectral region via complex Robert-Bonamy theory. J Mol Spectrosc 2007;243:113-123.

[60] Laraia A, Gamache RR, Hartmann JM, Perrin A, Gomez L. Theoretical calculations of $\mathrm{N}_{2}$-broadened half-widths of $\nu_{5}$ transitions of $\mathrm{HNO}_{3}$. J Quant Spectrosc Radiat Transfer 2009;110:687-699.

[61] Gabard T, Champion JP. Calculation of collision induced energy transfer rates in tetrahedral molecules. Application to ${ }^{12} \mathrm{CH}_{4}$ perturbed by argon. J Quant Spectrosc Radiat Transfer 1994;52:303-317.

[62] Gabard T. Argon-broadened Line Parameters in the $\nu_{3}$ Band of ${ }^{12} \mathrm{CH}_{4}$. J Quant Spectrosc Radiat Transfer 1997;57:177-196.

[63] Gabard T. Calculated Helium-broadened Line Parameters in the $\nu_{4}$ Band of ${ }^{13} \mathrm{CH}_{4}$. J Quant Spectrosc Radiat Transfer 1998;59:287-302.

[64] Tsao CJ, Curnutte B. Line widths of pressure-broadened spectral lines. J Quant Spectrosc Radiat Transfer 1962;2:41-91.

[65] Féjard L, Gabard T, Champion JP. Calculated Line Broadening Coefficients in the $\nu_{2}$ Band of $\mathrm{CH}_{3} D$ Perturbed by Helium. J Mol Spectrosc 2003;219:88-97.

[66] Tipping RH, Brown A, Ma Q, Hartmann JM, Boulet C, Liévin J. Collision-induced absorption in the $\nu_{2}$ fondamental band of $\mathrm{CH}_{4}$. I. Determination of the quadrupole transition moment. J Chem Phys 2001;115:8852-8857. 
[67] Hartmann JM, Brodbeck C, Flaud PM, Tipping RH, Brown A, Ma Q, et al. Collision-induced absorption in the $\nu_{2}$ fondamental band of $\mathrm{CH}_{4}$. II. Dependence on the perturber gas. J Chem Phys 2002;116:123-127.

[68] Kubo R. generalized cumulant expansion method. J Phys Soc Jap 1962;17:1100-1120.

[69] Kubo R. Stochastic Liouville equations. J Math Phys 1963;4:174-183.

[70] Ma Q, Tipping RH, Boulet C. Irreducible correlation functions of the $\widehat{S}$ matrix in the coordinate representation: Application in calculating Lorentzian half-widths and shifts. J Chem Phys 2006;124:014109.

[71] Ma Q, Tipping RH, Boulet C. Modification of the Robert-Bonamy formalism in calculating Lorentzian half-widths and shifts. J Quant Spectrosc Radiat Transfer 2007;103:588-596.

[72] Ma Q, Tipping RH, Boulet C, Thibault F, Bonamy J. Vibrationdependent trajectories and their effects on vibrational dephasing. J Mol Spectrosc 2007;243:105-112.

[73] The Symbolic Math Toolbox, The MathWorks, Inc. http://www . mathworks. com/products/symbolic/.

[74] Perl Programming Documentation. http://perldoc.perl.org/.

[75] Comprehensive Perl Archive Network. http://www. cpan.org/.

[76] Wenger C, Boudon V, Rotger M, Sanzharov M, Champion JP. XTDS and SPVIEW: Graphical tools for the analysis and simulation of highresolution molecular spectra. J Mol Spectrosc 2008;251:102-113.

[77] Bouanich JP, Blanquet G. Pressure broadening of CO and OCS spectral lines. J Quant Spectrosc Radiat Transfer 1988;40:205-220.

[78] Crovisier J. Constants for molecules of astrophysical interest in the gas phase: photodissociation, microwave and infrared spectra. http://www.lesia.obspm.fr/perso/jacques-crovisier/basemole/. 
[79] Gray CG, Gubbins KE. Theory of molecular fluids. Volume 1 : Fundamentals. Oxford: Clarendon Press; 1984.

[80] Pine AS. Self, $\mathrm{N}_{2}, \mathrm{O}_{2}, \mathrm{H}_{2}$, Ar and He-broadening in the $\nu_{3}$ band Q branch of $\mathrm{CH}_{4}$. J Chem Phys 1992;97:773-785.

[81] Rautian SG, Sobel'man II. The effect of collisions on the Doppler broadening of spectral lines. Sov Phys Usp 1967;9:701-716.

[82] Galatry L. Simultaneous effect of Doppler and foreign gas broadening on spectral lines. Phys Rev 1961;122:1218-1223.

[83] Yamamoto G, Hirono M. Rotational line width of methane. J Quant Spectrosc Radiat Transfer 1971;11:1537-1545.

[84] Tejwani GDT, Varanasi P. Calculation of collision-broadened linewidths in the infrared bands of methane. J Chem Phys 1971;55:1075-1083.

[85] Tejwani GDT, Fox K. Calculated linewidths for $\mathrm{CH}_{4}$ broadened by $\mathrm{N}_{2}$ and $\mathrm{O}_{2}$. J Chem Phys 1974;60:2021-2026.

[86] Varanasi P. Collision-broadened line widths of tetrahedral moleculesI. Theoretical formulation. J Quant Spectrosc Radiat Transfer 1974;14:995-1008.

[87] Tejwani GDT, Varanasi P, Fox K. Collision-broadened linewidths of tetrahedral molecules - II. Computations for $\mathrm{CH}_{4}$ lines broadened by $\mathrm{N}_{2}, \mathrm{O}_{2}, \mathrm{He}$, Ne and Ar. J Quant Spectrosc Radiat Transfer 1975;15:243254.

[88] Varanasi P. Collision-broadened linewidths of tetrahedral moleculesIII. Dispersion and induction interactions. J Quant Spectrosc Radiat Transfer 1975;15:255-258.

[89] Kalugina YN, Cherepanov VN, Buldakov MA, Zvereva-Loete N, Boudon V. Theoretical investigation of the potential energy surface of the van der Waals complex $\mathrm{CH}_{4}-\mathrm{N}_{2}$. J Chem Phys 2009;131:134304.

[90] Luo C, Wehr R, Drummond JR, May AD, Thibault F, Boissoles J, et al. Shifting and broadening in the fundamental band of CO highly diluted 
in He and Ar: A comparison with theory. J Chem Phys 2001;115:21982206.

[91] Thibault F, Martinez RZ, Domenech JL, Bermejo D, Bouanich JP. Raman and infrared linewidths of CO in Ar. J Chem Phys 2002;117:25232531.

[92] Cappelletti D, Bartolomei M, Carmona-Novillo E, Pirani F, Blanquet $\mathrm{G}$, Thibault F. Intermolecular interaction potentials for the $\mathrm{Ar}-\mathrm{C}_{2} \mathrm{H}_{2}$, $\mathrm{Kr}-\mathrm{C}_{2} \mathrm{H}_{2}$, and $\mathrm{Xe}-\mathrm{C}_{2} \mathrm{H}_{2}$ - weakly bound complexes: Information from molecular beam scattering, pressure broadening coefficients, and rovibrational spectroscopy. J Chem Phys 2007;126:064311.

[93] Gomez L, Martinez RZ, Bermejo D, Thibault F, Joubert P, BusseryHonvault B, et al. Q-branch linewidths of $\mathrm{N}_{2}$ perturbed by $\mathrm{H}_{2}$ : Experiments and quantum calculations from an ab initio potential. J Chem Phys 2007;126:204302.

[94] Thibault F, Fuller EP, Grabow KA, Hardwick JL, Marcus CI, Marston D, et al. Experimental line broadening and line shift coefficients of the acetylene $\nu_{1}+\nu_{3}$ band pressurized by hydrogen and deuterium and comparison with calculations. J Mol Spectrosc 2009;256:17-27.

[95] Bonamy J, Robert D, Boulet C. Simplified models for the temperature dependence of linewidths at elevated temperature and application to $\mathrm{CO}$ broadened by $\mathrm{Ar}$ and $\mathrm{N}_{2}$. J Quant Spectrosc Radiat Transfer 1984;31:23-34.

[96] Bauer A, Godon M, Kheddar M, Hartmann JM, Bonamy J, Robert D. Temperature and perturber dependences of water-vapor $380 \mathrm{GHz}$-line broadening. J Quant Spectrosc Radiat Transfer 1987;37:531-539.

[97] Gabard T, Grigoriev IM, Grigorovich NM, Tonkov MV. Helium and argon line broadening in the $\nu_{2}$ band of $\mathrm{CH}_{4}$. J Mol Spectrosc 2004;225:123-131.

[98] The CH4@Titan project. http://icb.u-bourgogne.fr/titan/.

[99] SpecMo - GDR CNRS 3152.

http://xeon.univ-reims.fr/specmo/. 
[100] Pôle de Sciences Planétaires Bourgogne Franche-Comté. http://www.icb.cnrs.fr/planeto/. 
Table 1: Lines of the $\nu_{4}$ band of methane with discrepencies larger than $50 \%$ for the line broadening coefficients

\begin{tabular}{|c|c|c|c|c|c|c|}
\hline $\begin{array}{c}\sigma_{\exp } \\
\left(\mathrm{cm}^{-1}\right)\end{array}$ & $\Delta \sigma^{a}$ & $\gamma_{\exp }^{b}$ & $\begin{array}{c}\Delta \gamma_{\exp }{ }^{c} \\
(\%) \\
\end{array}$ & $\gamma_{\text {calc }}{ }^{b}$ & $\begin{array}{c}\Delta \gamma^{d} \\
(\%)\end{array}$ & $\begin{array}{c}\operatorname{Branch}\left(J^{\prime \prime}\right), \\
C^{\prime \prime}, \alpha^{\prime \prime}, \alpha^{\prime} e^{-}\end{array}$ \\
\hline 1170.90030 & 0.033 & 0.01770 & 0.6 & 0.03992 & -125.5 & $\mathrm{P}(17), E, 3,6$ \\
\hline 1181.52849 & 0.100 & 0.01840 & 0.0 & 0.03997 & -117.2 & $\mathrm{P}(16), E, 3,6$ \\
\hline 1199.87443 & -0.510 & 0.01770 & 1.1 & 0.04386 & -147.8 & $\mathrm{P}(18), E, 1,9$ \\
\hline 1201.64092 & 0.112 & 0.02650 & 0.0 & 0.04381 & -65.3 & $\mathrm{P}(14), E, 3,5$ \\
\hline 1205.92670 & 0.049 & 0.02750 & 0.0 & 0.04525 & -64.6 & $\mathrm{P}(17), F_{2}, 1,12$ \\
\hline 1205.93045 & 0.077 & 0.02750 & 0.0 & 0.04522 & -64.5 & $\mathrm{P}(17), F_{1}, 1,13$ \\
\hline 1211.32963 & 0.206 & 0.02680 & 0.0 & 0.04342 & -62.0 & $\mathrm{P}(13), E, 2,5$ \\
\hline 1211.96704 & 3.538 & 0.01930 & 1.6 & 0.04464 & -131.3 & $\mathrm{P}(16), E, 1,8$ \\
\hline 1217.98304 & 0.239 & 0.03070 & 0.3 & 0.04617 & -50.4 & $\mathrm{P}(15), F_{2}, 1,11$ \\
\hline 1217.99560 & 0.178 & 0.02900 & 0.3 & 0.04611 & -59.0 & $\mathrm{P}(15), F_{1}, 1,11$ \\
\hline 1223.99463 & 0.204 & 0.02730 & 0.4 & 0.04677 & -71.3 & $\mathrm{P}(14), F_{2}, 1,10$ \\
\hline 1275.94654 & 0.883 & 0.02310 & 0.4 & 0.04618 & -99.9 & $\mathrm{Q}(15), F_{1}, 1,4$ \\
\hline 1279.66774 & -0.339 & 0.02640 & 1.5 & 0.04680 & -77.3 & $\mathrm{Q}(14), F_{2}, 1,4$ \\
\hline 1372.90110 & 0.018 & 0.02440 & 0.4 & 0.04274 & -75.2 & $\mathrm{R}(13), E, 2,1$ \\
\hline 1385.44060 & 0.097 & 0.01900 & 0.5 & 0.03987 & -109.8 & $\mathrm{R}(16), E, 3,1$ \\
\hline 1387.98719 & 0.111 & 0.02860 & 0.3 & 0.04563 & -59.5 & $\mathrm{R}(15), F_{2}, 1,4$ \\
\hline 1387.98933 & -0.384 & 0.02860 & 0.3 & 0.04558 & -59.4 & $\mathrm{R}(15), F_{1}, 1,4$ \\
\hline 1389.54369 & 0.239 & 0.02110 & 0.9 & 0.04135 & -96.0 & $\mathrm{R}(17), E, 3,1$ \\
\hline 1407.27456 & 0.049 & 0.01290 & 1.6 & 0.04430 & -243.4 & $\mathrm{R}(19), F_{2}, 1,5$ \\
\hline \multicolumn{7}{|c|}{$\begin{array}{l}a \sigma_{\exp }-\sigma_{\text {calc }}, \text { in } 10^{-3} \mathrm{~cm}^{-1} . \\
b \text { in } \mathrm{cm}^{-1} \cdot \text { atm }^{-1} \text {. } \\
c \text { Standard deviation from the fits, see Ref. [42]. } \\
d\left(\gamma_{\text {exp }}-\gamma_{\text {calc }}\right) / \gamma_{\text {exp }} .\end{array}$} \\
\hline
\end{tabular}



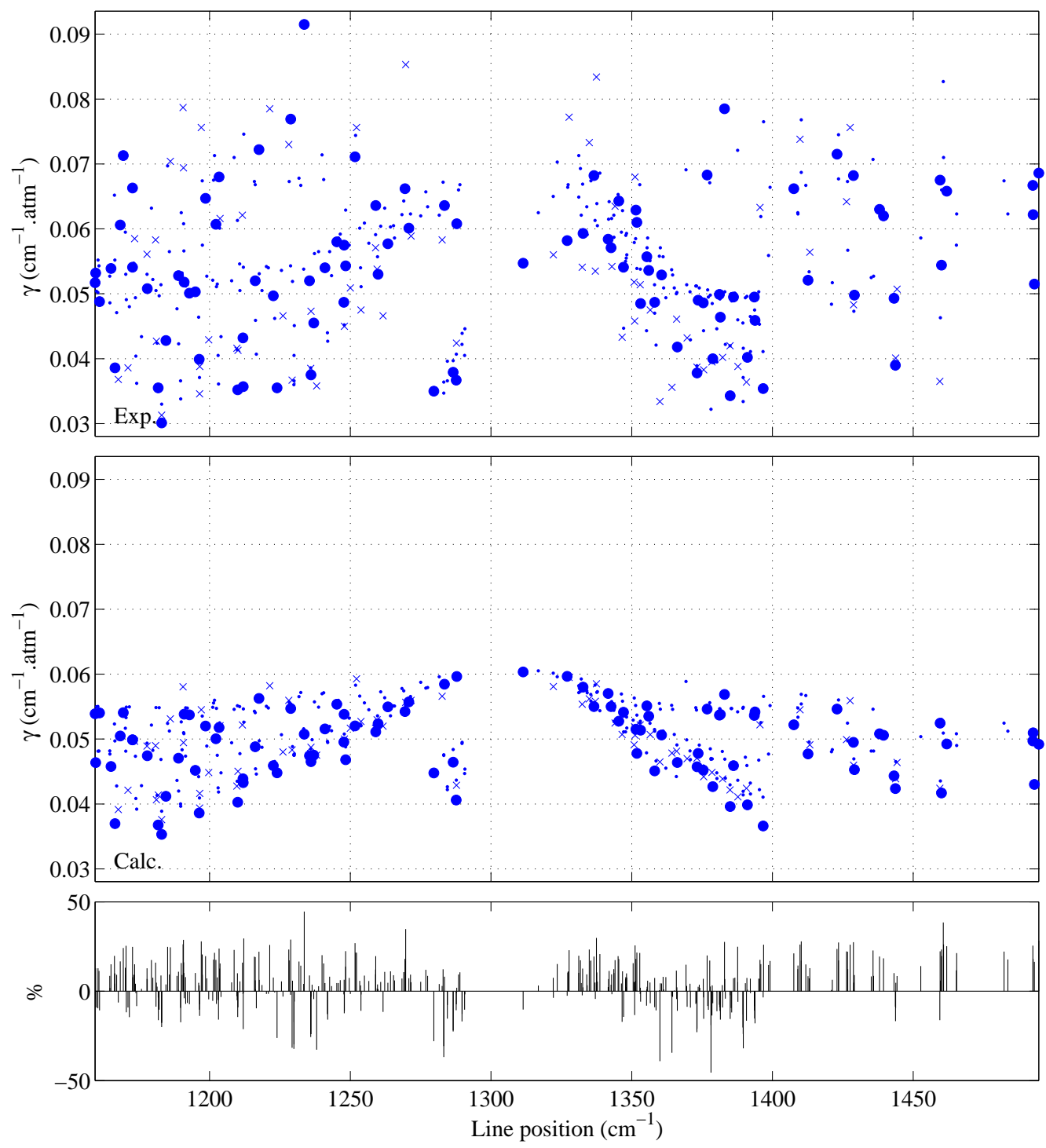

Figure 1: Observed (upper panel, from Ref. [42]) and calculated (middle panel) line broadening coefficients in the $\nu_{4}$ band of methane at room temperature. The perturber is air for the measurements and nitrogen for the calculations. Symbols are $\bullet$ for $A$ symmetries, $\times$ for $E$ symmetries and · for $F$ symmetries. Differences are shown on the lower panel. 


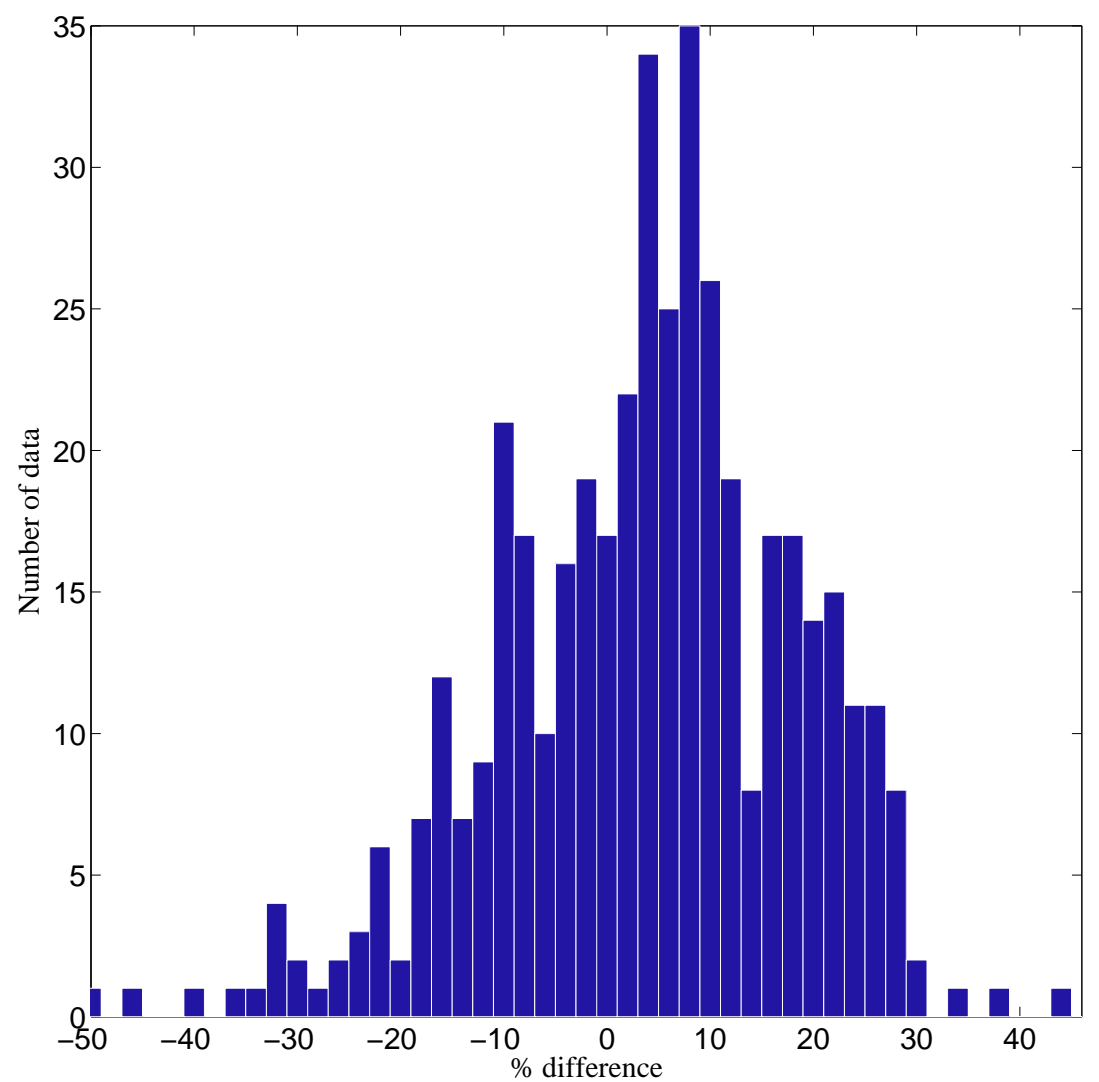

Figure 2: Histogram of the differences between observations and calculations for the data of Fig. 1 ( $\nu_{4}$ band). 

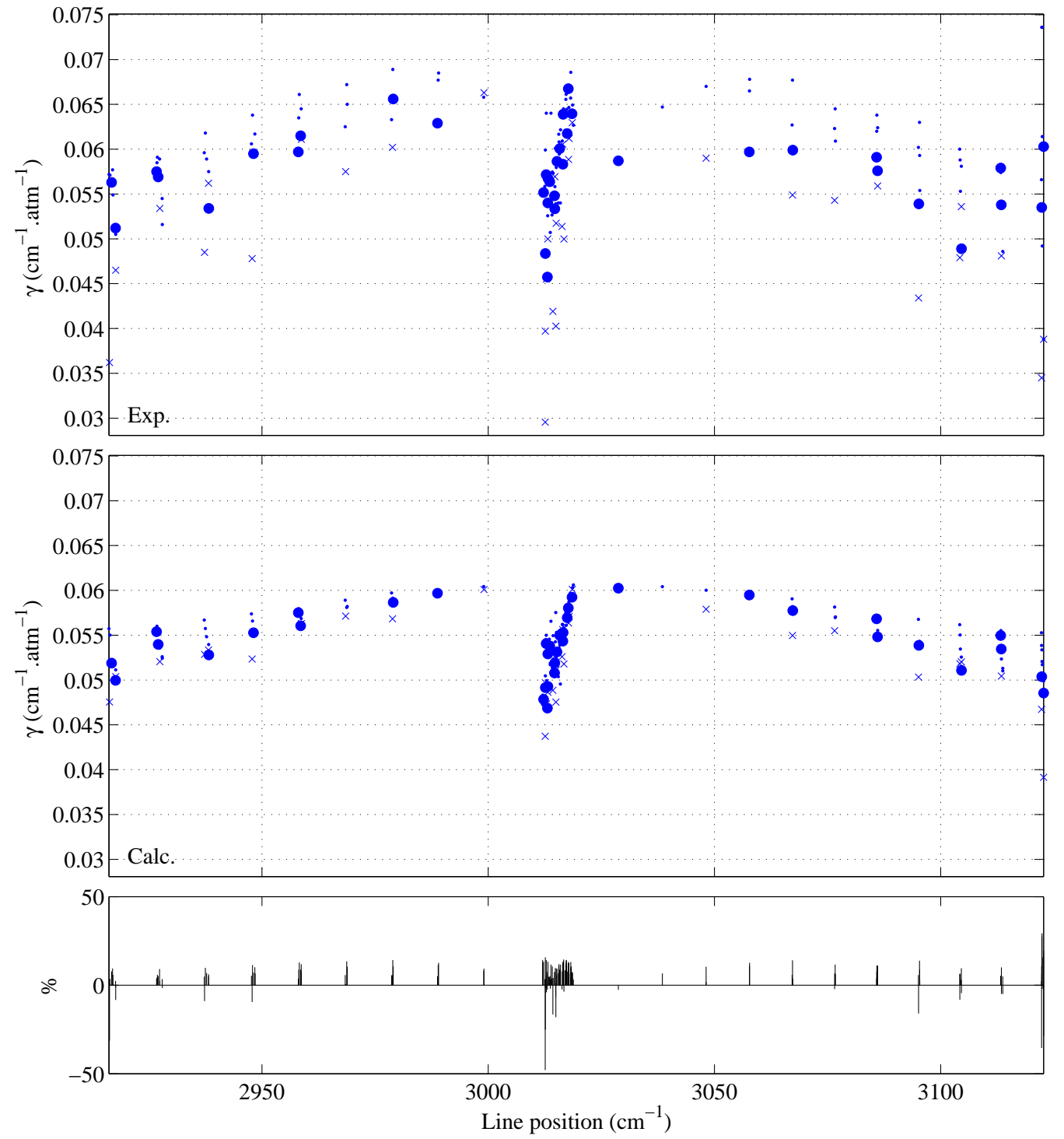

Figure 3: Observed (upper panel, from Refs. [47, 52]) and calculated (middle panel) nitrogen line broadening coefficients in the $\nu_{3}$ band of methane at room temperature. Symbols are $\bullet$ for $A$ symmetries, $\times$ for $E$ symmetries and $\cdot$ for $F$ symmetries. Differences are shown on the lower panel. 

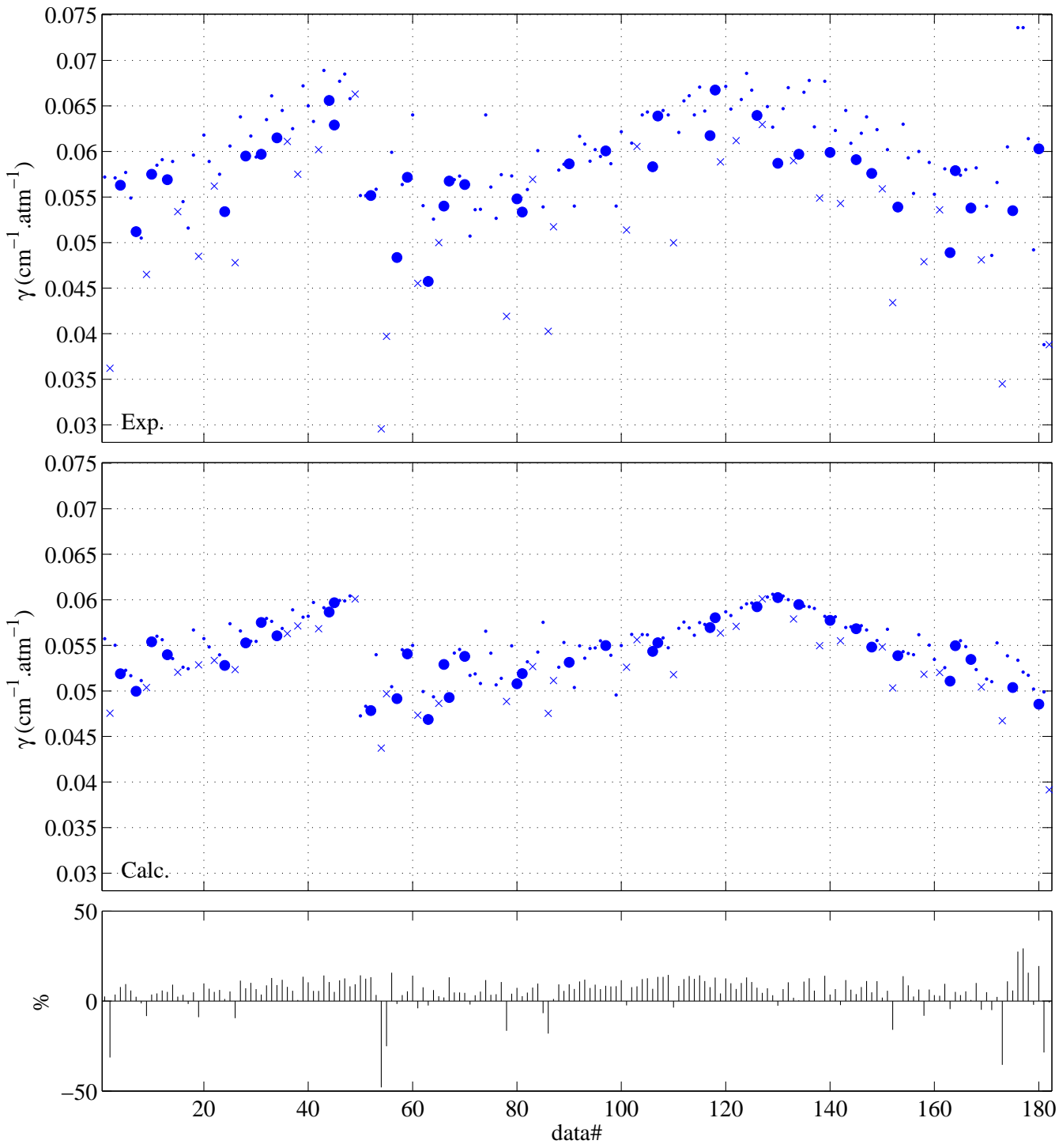

Figure 4: Same as Fig. 3, except that data are plotted vs the running indices of the lines (increasing wavenumber order). Data\# from 1 to 49 : P branch, Data\# from 50 to 129 : Q branch, Data\# from 130 to 182 : R branch. 


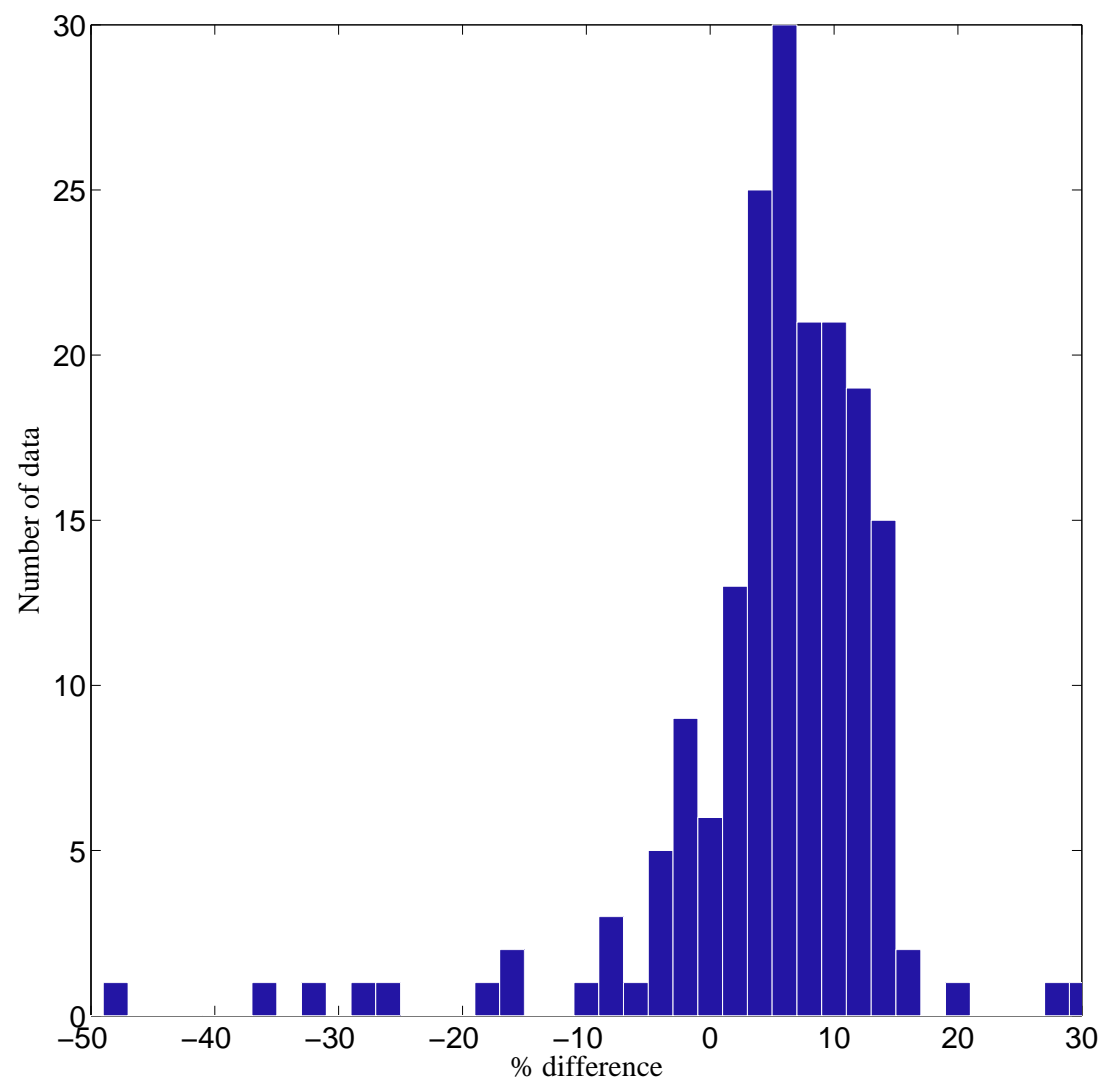

Figure 5: Histogram of the differences between observations and calculations for the data of Figs. 3 and 4 ( $\nu_{3}$ band). 

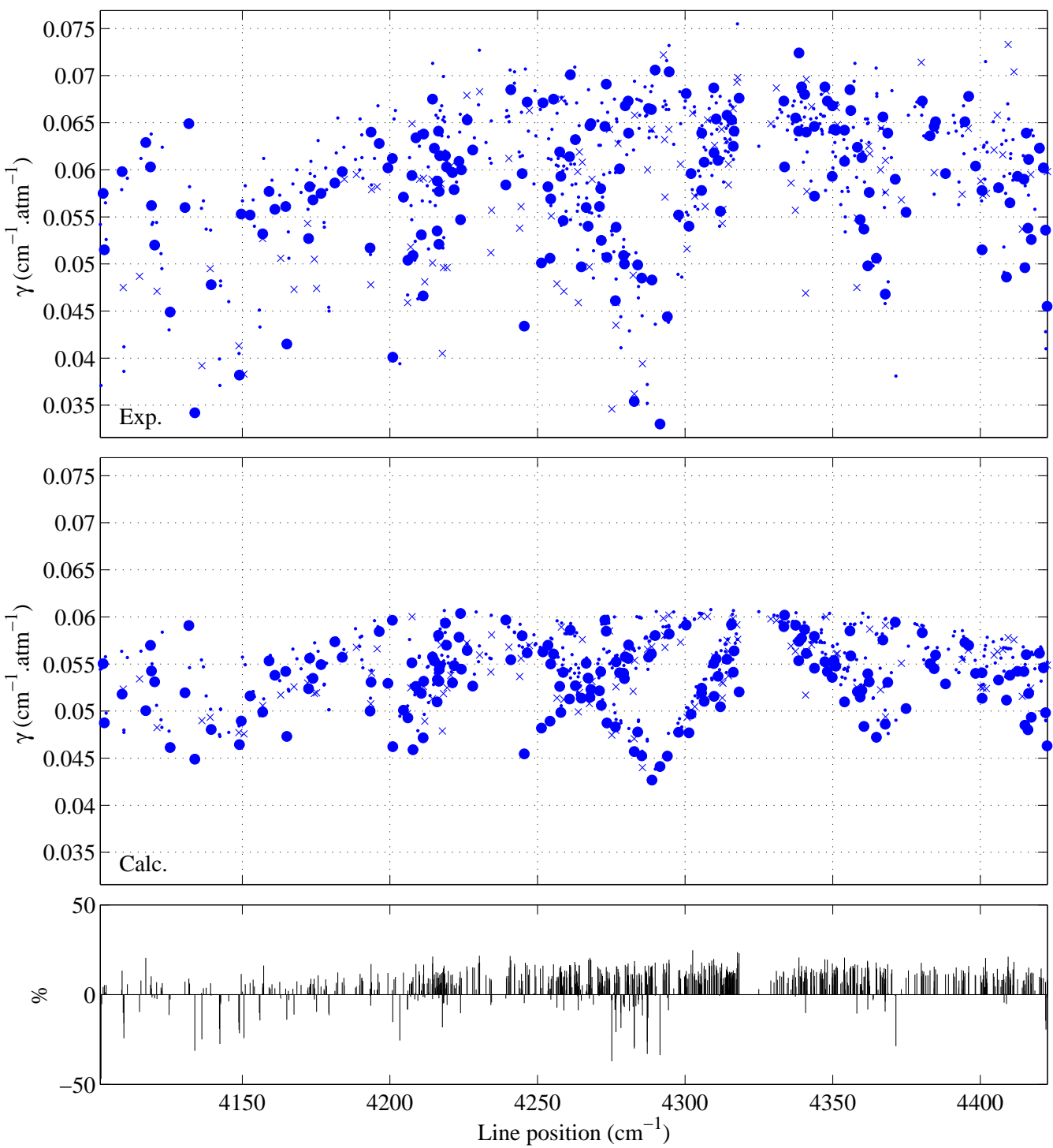

Figure 6: Observed (upper panel, from Ref. [34]) and calculated (middle panel) line broadening coefficients in the octad region of methane at room temperature. The perturber is air for the measurements and nitrogen for the calculations. Symbols are $\bullet$ for $A$ symmetries, $\times$ for $E$ symmetries and $\cdot$ for $F$ symmetries. Differences are shown on the lower panel. 


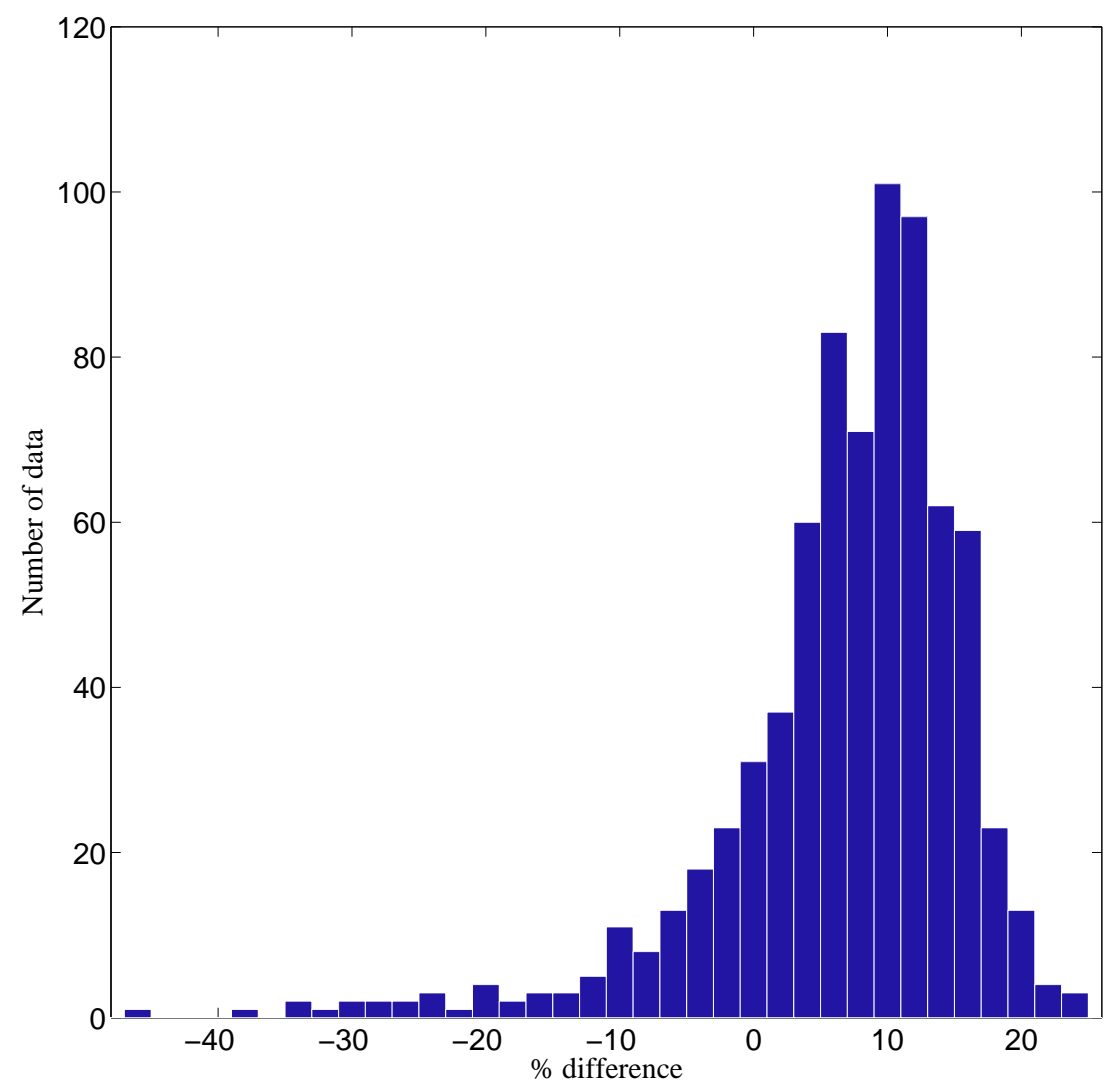

Figure 7: Histogram of the differences between observations and calculations for the data of Fig. 6 (octad region). 